\title{
Incorporating HYPR de-noising within iterative PET reconstruction (HYPR-OSEM)
}

DOI:

10.1088/1361-6560/aa7b66

Document Version

Accepted author manuscript

Link to publication record in Manchester Research Explorer

\section{Citation for published version (APA):}

Cheng, J-C., Matthews, J., Sossi, V., Anton-Rodriguez, J., Salomon, A., \& Boellaard, R. (2017). Incorporating HYPR de-noising within iterative PET reconstruction (HYPR-OSEM). Physics in Medicine and Biology. https://doi.org/10.1088/1361-6560/aa7b66

\section{Published in:}

Physics in Medicine and Biology

\section{Citing this paper}

Please note that where the full-text provided on Manchester Research Explorer is the Author Accepted Manuscript or Proof version this may differ from the final Published version. If citing, it is advised that you check and use the publisher's definitive version.

\section{General rights}

Copyright and moral rights for the publications made accessible in the Research Explorer are retained by the authors and/or other copyright owners and it is a condition of accessing publications that users recognise and abide by the legal requirements associated with these rights.

\section{Takedown policy}

If you believe that this document breaches copyright please refer to the University of Manchester's Takedown Procedures [http://man.ac.uk/04Y6Bo] or contact uml.scholarlycommunications@manchester.ac.uk providing relevant details, so we can investigate your claim.

\section{OPEN ACCESS}


Incorporating HYPR de-noising within iterative PET reconstruction (HYPR-OSEM)

This content has been downloaded from IOPscience. Please scroll down to see the full text.

Download details:

IP Address: 130.88.240.90

This content was downloaded on 29/06/2017 at 14:20

Manuscript version: Accepted Manuscript

Cheng et al

To cite this article before publication: Cheng et al, 2017, Phys. Med. Biol., at press: https://doi.org/10.1088/1361-6560/aa7b66

This Accepted Manuscript is: () 2017 Institute of Physics and Engineering in Medicine

During the embargo period (the 12 month period from the publication of the Version of Record of this article), the Accepted Manuscript is fully protected by copyright and cannot be reused or reposted elsewhere.

As the Version of Record of this article is going to be / has been published on a subscription basis, this Accepted Manuscript is available for reuse under a CC BY-NC-ND 3.0 licence after the 12 month embargo period.

After the embargo period, everyone is permitted to copy and redistribute this article for non-commercial purposes only, provided that they adhere to all the terms of the licence https://creativecommons.org/licences/by-nc-nd/3.0

Although reasonable endeavours have been taken to obtain all necessary permissions from third parties to include their copyrighted content within this article, their full citation and copyright line may not be present in this Accepted Manuscript version. Before using any content from this article, please refer to the Version of Record on IOPscience once published for full citation and copyright details, as permission will likely be required. All third party content is fully copyright protected, unless specifically stated otherwise in the figure caption in the Version of Record.

When available, you can view the Version of Record for this article at: http://iopscience.iop.org/article/10.1088/1361-6560/aa7b66 


\section{Incorporating HYPR de-noising within iterative PET reconstruction (HYPR-OSEM)}

Ju-Chieh (Kevin) Cheng ${ }^{1,2,3,4}$, Julian Matthews ${ }^{2}$, Vesna Sossi ${ }^{3}$, Jose Anton-Rodriguez ${ }^{2}$, André Salomon ${ }^{5}$, and Ronald Boellaard ${ }^{1,6}$

${ }^{1}$ Department of Radiology and Nuclear Medicine, VU University Medical Center, De Boelelaan 1117, Amsterdam, $1081 \mathrm{HV}$, NL

${ }^{2}$ Division of Informatics, Imaging and Data Sciences, The University of Manchester, Wolfson Molecular Imaging Centre, Manchester M20 3LJ, UK

${ }^{3}$ Department of Physics and Astronomy, The University of British Columbia, 6224 Agricultural Road, Vancouver, B.C. V6T 1Z1, Canada

${ }^{4}$ Pacific Parkinson's Research Centre, The University of British Columbia, 2215 Wesbrook Mall, Vancouver, B.C. V6T 1Z3, Canada

${ }^{5}$ Department of Oncology Solutions, Philips Research, HTC 34, Eindhoven, 5656 AE, NL

${ }^{6}$ Department of Nuclear Medicine and Molecular Imaging, University Medical Center Groningen, University of Groningen, Hanzeplein 1, 9713 KC, Groningen, NL

* Corresponding author

E-mail: chengjuchieh@gmail.com (JC)

Short title: Incorporating HYPR de-noising within OSEM 


\section{Abstract}

HighlY constrained back-PRojection (HYPR) is a post-processing de-noising technique originally developed for time-resolved Magnetic Resonance Imaging. It has been recently applied to dynamic imaging for Positron Emission Tomography (PET) and shown promising results. In this work, we have developed an iterative reconstruction algorithm (HYPR-OSEM) which improves the signal-to-noise ratio (SNR) in static imaging (i.e. single frame reconstruction) by incorporating HYPR de-noising directly within the ordered subsets expectation maximization (OSEM) algorithm. The proposed HYPR operator in this work operates on the target image(s) from each subset of OSEM and uses the sum of the preceding subset images as the composite which is updated every iteration. Three strategies were used to apply the HYPR operator in OSEM: i) within the image space modeling component of the system matrix in forward-projection only, ii) within the image space modeling component in both forward-projection and back-projection, and iii) on the image estimate after the OSEM update for each subset thus generating 3 forms: i) HYPR-F-OSEM, ii) HYPR-FB-OSEM, and iii) HYPR-AU-OSEM. Resolution and contrast phantom simulations with various sizes of hot and cold regions as well as experimental phantom and patient data were used to evaluate the performance of the 3 forms of HYPR-OSEM, and the results were compared to OSEM with and without a post reconstruction filter. It was observed that the convergence in contrast recovery coefficients (CRC) obtained from all forms of HYPR-OSEM was slower than that obtained from OSEM. Nevertheless, HYPR-OSEM improved SNR without degrading accuracy in terms of resolution and contrast. It achieved better accuracy in CRC at equivalent noise level and better precision than OSEM and better accuracy than filtered OSEM in general. In addition, HYPR-AU-OSEM has been determined to be the more effective form of HYPR-OSEM in terms of accuracy and precision based on the studies conducted in this work. 


\section{Introduction}

Reconstructed images for Positron Emission Tomography (PET) are generally noisy compared to those obtained from other medical imaging techniques such as Magnetic Resonance Imaging (MRI) and Computed Tomography (CT) since the image reconstruction problem is ill-conditioned, and the quality of PET images is highly dependent on the severely limited number of coincidence events acquired. Although single frame static imaging usually acquires more counts per frame thus corresponding to a higher signal-to-noise ratio (SNR) than a single dynamic frame, the SNR of typical static PET scans is not always satisfactory. For example, lesion detection and studies of tumor heterogeneity (Vriens et al. 2012, Nyflot et al. 2015) are highly sensitive to noise. Furthermore, in clinical practice, low dose and/or short scan time acquisitions are preferred especially in pediatric imaging (Boellaard et al. 2010). For large patients, photon attenuation significantly degrades the SNR. Although the dose can be increased, this is typically insufficient to make up for the SNR loss. A low number of acquired counts is also common when imaging is performed using unconventional radioisotopes with a low positron branching fraction, for instance, yttrium-90 (Fowler et al. 2016). Therefore, further improvement in SNR within a single frame PET reconstruction is highly desirable, with single frame improvements also relevant to multi-frame dynamic PET imaging.

Various methods have been proposed to improve the SNR in dynamic PET imaging such as 4D PET reconstruction (Rahmim et al. 2009, Reader and Verhaeghe 2014) and HighlY constrained back-PRøjection (HYPR) post processing (Johnson et al. 2008, Christian et al. 2010, Floberg et al. 2012). For static imaging, regularization techniques such as post reconstruction filtering and prior based methods have been proposed over the years (Bai et al. 2013). However, the prior based methods generally require the definition of a prior and taskspecific parameters before the reconstruction. As a result, they have not been widely used 
clinically. The current gold standard method for improving the SNR in static imaging is applying (Gaussian) filtering due to its fast performance and ease of implementation. Moreover, it does not require any prior knowledge of the imaging task. On the other hand, it achieves noise reduction at the cost of image resolution and contrast. In this work, HYPR denoising was directly incorporated within the widely used Ordered Subset Expectation Maximization (OSEM) algorithm (Hudson and Larkin 1994) to improve the SNR for PET without degrading accuracy in terms of resolution and contrast. Furthermore, the proposed method is not computationally intensive and does not require any prior information. The performance of various forms of HYPR-OSEM in static imaging was evaluated using resolution and contrast phantom simulations as well as using experimental phantom and patient studies.

\section{Methods}

HYPR de-noising

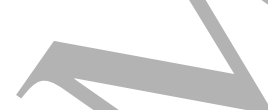

HYPR post processing is a promising de-noising technique originally developed for time-resolved MRI, and it has been further refined by constraining the back-projections to local regions of interest (HYPR-LR). The HYPR-LR method (Johnson et al. 2008) is given by:

$$
H_{t}=C_{t} \cdot \frac{F * I_{t}}{F * C_{t}}
$$

where $H_{t}$ is the HYPR de-noised image for the $t^{\text {th }}$ time frame of the dynamic series, $I_{t}$ is the target image at $t^{\text {th }}$ time frame, $C_{t}$ is the composite image for the $t^{\text {th }}$ time frame, $F$ is the filter function, and * represents the convolution operation. The composite image is a high resolution and less noisy image than the target, and it is typically defined as the weighted sum 
of dynamic frame images according to the frame duration for the case of PET. From Eq.(1), one can observe that the noise in the HYPR de-noised image is contributed from both the composite and the filtered target. Although the noise textures in MR images may be quite different from those in PET, the idea of using high frequency components of the composite to preserve resolution and to constrain noise in the target is still applicable to PET and other imaging modality such as CT. Consequently, HYPR-LR post processing has been recently applied to dynamic PET and shown promising results in improving SNR (Christian et al. 2010, Floberg et al. 2012).

\section{HYPR-OSEM}

The OSEM algorithm used here is given by:

$$
\begin{aligned}
& \lambda_{j}^{m, l}=\frac{\lambda_{j}^{m, l-1}}{\sum_{i \in s_{l}} P_{i j}} \sum_{i \in s_{l}} P_{i j} \frac{y_{i}}{\sum_{j=1}^{J} P_{i j} \lambda_{j}^{m, l-1}} \\
& \lambda_{j}^{m, 0}=\left\{\begin{array}{c}
1.0 \text { if } m=0 \\
\lambda_{j}^{m-1, L} \text { if } m>0
\end{array}\right. \\
& P_{i j}=W_{i i} G_{i j} B_{j j}
\end{aligned}
$$

where $y_{i}$ is the measured projection data along the $i^{\text {th }}(i=1, \ldots \ldots, \mathrm{I})$ line-of-response (LOR) and $\lambda_{j}^{m, l}$ is the image intensity estimate in voxel $j(j=1, \ldots \ldots, \mathrm{J})$ at the $l^{\text {th }}(l=1$ subset $\left(S_{l}\right)$ of $m^{\text {th }}$ iteration. $P \in R^{I \times J}$ is the system matrix which typically consists of 3 parts: a) a weighting component ( $W \in R^{I \times I}$ ) which accounts for normalization and attenuation corrections (detector blurring effect can also be modeled here), b) a geometrical component ( $G \in R^{I \times J}$, which provides a mapping between the LOR's or bins of the sinograms and the image voxels, and c) an image-space modeling component $\left(B \in R^{J \rtimes J}\right)$ which, for example, could represent resolution and object motion blurring. 
The proposed HYPR de-noising operator in this work is defined as:

$$
\begin{aligned}
& H_{m}\left(I_{j}^{m, l}\right)=H_{C_{j}^{m} ; F}\left(I_{j}^{m, l}\right)=C_{j}^{m} \cdot \frac{F * I_{j}^{m, l}}{F * C_{j}^{m}} \text {, where } \\
& C_{j}^{m}=\sum_{l} I_{j}^{m-1, l}
\end{aligned}
$$

where $I_{j}^{m, l}$ is the target image at $l^{\text {th }}$ subset of $m^{\text {th }}$ iteration, $C_{j}^{m}$ is the composite at $m^{\text {th }}$ iteration, $F$ is the filter function (Gaussian in this case), and * represents the convolution operation. The composite here is updated for each OSEM iteration as the sum of the preceding subset images from the previous iteration; i.e the composite is updated once per iteration after the final subset update of each OSEM iteration loop. To prevent singularity issues caused by dividing by zero, a small value (i.e. $10^{-10}$ in this case) was assigned to the HYPR de-noised image for voxels with zero value in the filtered composite. Note that when applying HYPR (post-processing) to dynamic imaging, the contrast in the composite needs to be very close to that in the target image to minimize bias introduced from the composite to the target (Floberg et al. 2012). For the case of directly incorporating HYPR within OSEM, HYPR is not expected to introduce any bias, other than changing the convergence speed, since the subset data correspond to the same tracer distribution, and the goal is to estimate this distribution. In summary, the proposed method makes use of the high frequency components from the composite to preserve resolution and to constrain noise in the target for each update of OSEM. Furthermore, the composite formation scheme proposed here can help avoid bias toward any particular OSEM subset as well as reducing the probability of having voxel value of zero within the object. Consequently, it allows effective update of contrast through the iterative update of the composite with a highly constrained noise increment per OSEM update. Ideally, the composite image in HYPR needs to have high resolution with low noise, and several initial OSEM updates are required to obtain a high resolution composite. On the 
other hand, noise increases as the number of updates increases. Here the HYPR operator (composite) was incorporated to constrain the noise increment after only one iteration of OSEM with 16 subsets in order to achieve a high SNR gain and to avoid high frequency noise in the composite.

Three strategies were used to incorporate the HYPR operator in OSEM: i) before Forward-projection, ii) before Forward-projection and after the Back-projection operations, and iii) After the OSEM subset Update (i.e. separated from the update factor) thus generating 3 forms of HYPR-OSEM. These three methods are denoted as HYPR-F-OSEM, HYPR-FBOSEM, and HYPR-AU-OSEM, respectively as shown in Eqs.(4-6).

i) HYPR-F-OSEM:

$$
\lambda_{j}^{m, l}=\frac{\lambda_{j}^{m, l-1}}{\sum_{i \in s_{l}} P_{i j}} \sum_{i \in s_{l}} P_{i j} \frac{y_{i}}{\sum_{j=1}^{J} P_{i j} H_{m}\left(\lambda_{j}^{m, l-1}\right)}
$$

ii) HYPR-FB-OSEM:

iii) HYPR-AU-OSEM:

$$
\lambda_{j}^{m, l}=\frac{\lambda_{j}^{m, l-1}}{H_{m}\left(\sum_{i \in s_{l}} P_{i j}\right)} H_{m}\left(\sum_{i \in s_{l}} P_{i j} \frac{y_{i}}{\sum_{j=1}^{J} P_{i j} H_{m}\left(\lambda_{j}^{m, l-1}\right)}\right)
$$

$$
\lambda_{j}^{m, l}=H_{m}\left(\frac{\lambda_{j}^{m, l-1}}{\sum_{i \in s_{l}} P_{i j}} \sum_{i \in s_{l}} P_{i j} \frac{y_{i}}{\sum_{j=1}^{J} P_{i j} \lambda_{j}^{m, l-1}}\right)
$$

$I_{j}^{m, l}$ in Eq.(3) can represent the activity image estimate, the update factor image, or the sensitivity image. Although Eqs.(4) and (6) are equivalent on the procedural level of 
application of the HYPR operator in the middle of the iterative process (i.e. applying the HYPR operator to the output image in Eq.(6) or to the input image in Eq.(4)), nevertheless HYPR-F-OSEM and HYPR-AU-OSEM differ in: a) HYPR-F-OSEM does not deal with the 'zero trapping' issue (Vaissier et al. 2013) very well as the zero trapping can always come back from the back-projection step, whereas HYPR-AU-OSEM can sufficiently reduce the zero trapping; b) the composite becomes different between HYPR-F-OSEM and HYPR-AUOSEM after the $2^{\text {nd }}$ iteration as each algorithm generates different subset images from the $2^{\text {nd }}$ iteration and on.

\section{$2 D$ simulations of resolution and contrast phantoms}

Feasibility of the proposed methods was first evaluated using simulations. Two phantoms, one consisting of 3 point sources and the other consisting of various sizes of hot and cold regions as well as a uniform background similar to the NEMA IQ phantom, were simulated. The diameter of the contrast phantom was $273 \mathrm{~mm}$, and the dimensions of the cold regions were 35 and $30 \mathrm{~mm}$ in diameter. The diameter of the hot regions ranged from $25 \mathrm{~mm}$ to $10 \mathrm{~mm}$, and a hot-to-background ratio of 4;1 was assigned. The virtual scanner projection data (sinogram) with 256 bins and 144 angular views were generated by forward-projecting the phantom images with an image matrix size of 256 x 256 and voxel size of $2.5 \mathrm{~mm}$. Prior to the forward-projection, the phantom images were smoothed with a $5 \mathrm{~mm}$ Gaussian filter to simulate the intrinsic resolution of a typical clinical PET scanner. The resultant resolution (FWHM) of the point source in the reference image was $5.5 \mathrm{~mm}$. Poisson noise was added to the simulated projection data. The effect of background contamination such as the scatter and randoms was not included in the simulations. The point source phantom was simulated with a high number of counts of 1 million while the contrast phantom was simulated under a lower counting condition $(100,000$ counts: $\sim 5$ counts per sinogram bin). The simulated projection 
data were reconstructed with all 3 forms of HYPR-OSEM, OSEM and filtered OSEM (5 mm

FWHM Gaussian post reconstruction filter) with 16 subsets and up to 16 iterations.
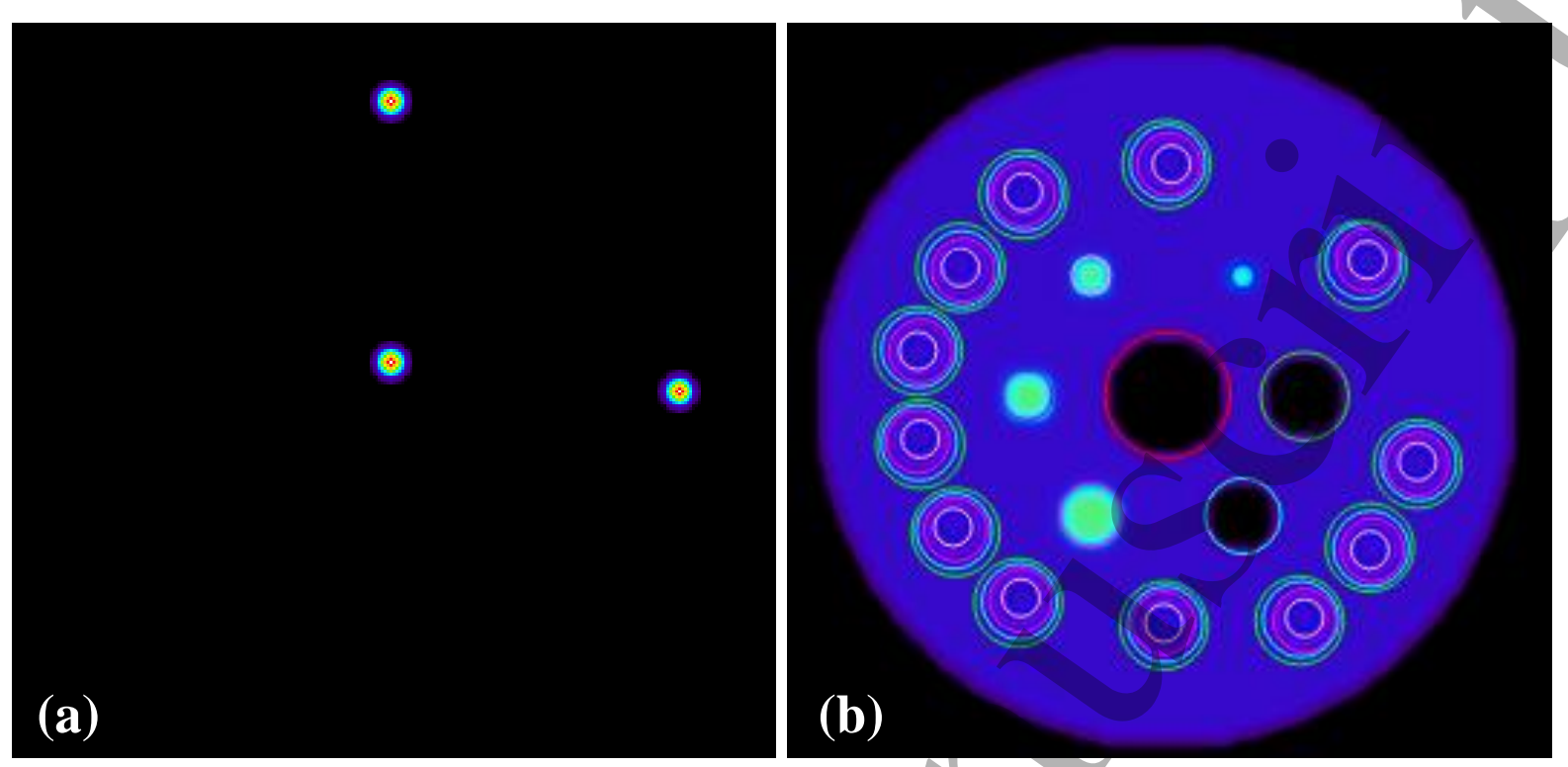

Figure 1. (a) The simulated point source phantom and (b) the contrast phantom with the ROI's used for the analysis according to the NEMA standard. Note that the biggest cold region at the center of the contrast phantom was not used in the evaluation. The ROI's which share the same color have the same size. All the ROI's placed on the uniform background of the phantom are used for noise evaluations, and their sizes match with the ROI sizes of the hot and cold regions according to the NEMA protocol.

The resolution versus iteration and contrast recovery coefficient (CRC) versus voxelnoise were evaluated and compared across all reconstruction methods. The resolution (both FWHM and FWTM) was measured as the average of the transverse resolution (average over both radial and tangential resolutions) over the 3 point sources. The voxel-noise in the reconstructed image is characterized by the coefficient of variation (COV) for voxels in uniform background regions. The simulated phantoms along with the regions of interest (ROI) used for the contrast and noise analysis are shown in figure 1. The NEMA standard (NEMA 2007) was used for both point source positioning and contrast phantom analyses. The CRC's for the hot and cold regions are defined in Eqs.(7) and (8), respectively.

$$
C R C_{h o t}=\left(\frac{\frac{C_{H}}{C_{B}}-1}{\frac{A_{H}}{A_{B}}-1}\right) \times 100 \%
$$




$$
C R C_{\text {cold }}=\left(1-\frac{C_{c}}{C_{B}}\right) \times 100 \%
$$

where $C_{B}, C_{H}$, and $C_{c}$ are the mean counts within the background, hot, and cold regions, respectively. $A_{H}$ and $A_{B}$ are the reference activities for the hot and background regions, respectively.

Filter function and evaluation of effect of filter kernel size

The filter function used in the HYPR operator was a Gaussian with a $5 \mathrm{~mm}$ FWHM for the simulations except for the early updates. Since the changes between the early updates are more drastic than those between the later updates, a narrower filter kernel (3 mm FWHM) was used for one iteration right after the initial OSEM iteration to limit the cross-talk between the composite and the target. For HYPR-FB-OSEM, a $3 \mathrm{~mm}$ kernel was also used for the HYPR operator in the back-projection throughout all iterations to ensure accurate update factors can be obtained for all cases; i.e. the update factors are less consistent across different subsets than the tracer distribution.

In addition to applying the $5 \mathrm{~mm}$ FWHM Gaussian filter kernel in the HYPR operator, $3 \mathrm{~mm}$ FWHM and $10 \mathrm{~mm}$ FWHM were used to evaluate the effect of filter kernel size for all forms of HYPR-OSEM using the same phantom simulations described above. As previously mentioned, a 3 mm FWHM kernel was always used in the HYPR operator for one iteration right after the initial OSEM iteration for all forms of HYPR-OSEM. The $3 \mathrm{~mm}$ kernel was also used for the HYPR operator in the back-projection for all cases of HYPR-FB-OSEM.

Coefficient of variation vs bias and percent root-mean-squared-error vs iteration

10 independent noisy realizations of the contrast phantom data as mentioned above were generated and reconstructed with all forms of HYPR-OSEM, OSEM, and OSEM 
filtered with a $5 \mathrm{~mm}$ Gaussian kernel to match with that used in the HYPR operator. The \% COV in CRC vs \%Bias in CRC (i.e. precision vs accuracy) and the percent root-meansquared-error (\%RMSE: a measure of both bias and variance) in CRC across all realizations was plotted for all sizes of hot and cold regions and compared across all reconstruction methods. The \%COV, \%Bias, and \%RMSE are defined in Eqs. (9-11).

$$
\begin{aligned}
& \% C O V_{C R C}=\left(\frac{1}{\overline{C R C}}\right) \sqrt{\frac{\sum_{n=1}^{N}\left(C R C_{n}-\overline{C R C}\right)^{2}}{N-1}} \times 100 \% \\
& \overline{C R C}=\frac{\sum_{n=1}^{N} C R C_{n}}{N} \\
& \% \operatorname{Bias}_{C R C}=\left(\frac{\overline{C R C}-C R C_{R}}{C R C_{R}}\right) \times 100 \% \\
& \% \operatorname{RMSE}_{C R C}=\left(\frac{1}{C R C_{R}}\right) \sqrt{\frac{\sum_{n=1}^{N}\left(C R C_{n}-C R C_{R}\right)^{2}}{N}} \times 100 \%
\end{aligned}
$$

where $C R C_{n}$ is the CRC obtained from the $n^{\text {th }}$ realization, $N$ is the total number of realizations, and $C R C_{R}$ is the reference $\mathrm{CRC}$ value.

\section{Experimental contrast phantom scan}

A modified plexiglass Esser phantom consists of a number of cylindrical inserts contained within a $204 \mathrm{~mm}$ cylindrical body. Each insert consists of a thin walled $38 \mathrm{~mm}$ long cylinder with varying diameters $(8,12,16$, and $25 \mathrm{~mm})$. The phantom was modified by replacing two of the $25 \mathrm{~mm}$ diameter cylinders, including the solid Telflon cylinder, with smaller $4 \mathrm{~mm}$ and $6 \mathrm{~mm}$ diameter cylinders but of equal length to the other cylinders. The phantom was filled with fluorine-18 $\left({ }^{18} \mathrm{~F}\right)$ with the hot-to-background activity ratio of 
approximately $4: 1$ for the $4,6,8,12$, and $16 \mathrm{~mm}$ diameter cylinders and with the $25 \mathrm{~mm}$ diameter cylinders containing cold (no activity) water. The total activity within the phantom was approximately $40 \mathrm{MBq}$ at the start of data acquisition with data recorded for 8 hours on the High Resolution Research Tomograph (HRRT) (Hugo et al. 2007). The list-mode data were histogrammed into span 9 (axial rebinning) sinograms.

50 independent realizations of the experimental phantom data were then generated using the bootstrap resampling method (Lartizien et al. 2010, Markiewicz et al. 2015) for two different count levels: one is approximately equivalent to a dynamic frame with a high number of counts ( 213 million) from a carbon-11 [ $\left.{ }^{11} \mathrm{C}\right] \mathrm{PK} 11195$ study, while the other corresponds to a dynamic frame with a low number of counts $(\sim 20$ million $)$ for the HRRT. The phantom data were then reconstructed using up to 50 iterations with 16 subsets, with all aforementioned reconstruction methods including all corrections (i.e. randoms, scatter, attenuation, and normalization), and using an image matrix size of 256 x 256 x 207 and an image voxel size of $1.22 \mathrm{~mm} \times 1.22 \mathrm{~mm} \times 1.22 \mathrm{~mm}$. For the experimental data, the kernel sizes were reduced by a factor of 2 in the HYPR/operator(s); i.e. $2.5 \mathrm{~mm}$ FWHM and $1.5 \mathrm{~mm}$ FWHM. This is because the intrinsic resolution and image voxel size for the HRRT are two times better/smaller than those used in the simulations, which correspond to those of typical clinical PET scanners. The $2.5 \mathrm{~mm}$ FWHM kernel was also used in the post reconstruction Gaussian filter for OSEM imalges. $1.5 \mathrm{~mm}$ FWHM kernel size was used during the $2^{\text {nd }}$ iteration of all HYPR-OSEM methods as well as in the HYPR operator of the back-projection for HYPR-FB-OSEM. Similar analyses as described above for the simulations were performed.

Clinical patient study 
A $\left[{ }^{11} \mathrm{C}\right] \mathrm{PK} 11195$ glioma patient study was conducted on the HRRT. $665 \mathrm{MBq}$ of PK11195 was injected, and list-mode emission data were acquired for 60 minutes. The listmode data were then histogrammed into 17 dynamic frames. The $1^{\text {st }}$ frame with 15 second frame duration $(\sim 20 \mathrm{M}$ counts $)$ and the $9^{\text {th }}$ frame with 300 second frame duration $(\sim 213 \mathrm{M}$ counts) were selected for low and high count evaluations of the proposed reconstruction method. The two frames were reconstructed with the number of iterations which achieves the lowest RMSE in CRC's according to the experimental contrast phantom study for OSEM, filtered OSEM, and HYPR-AU-OSEM.

\section{Results \\ $2 D$ resolution and contrast phantom simulations}

The figures (S1-S7) corresponding to the simulation results can be found in the supplementary data. The FWHM and FWTM as a function of iterations are shown in figure S1. The resolution performances for all forms of HYPR-OSEM were comparable to OSEM, whereas the resolution obtained from the filtered OSEM was $\sim 7.5 \mathrm{~mm}$ FWHM and $\sim 13.5 \mathrm{~mm}$ FWTM (not shown). Small variation in resolution was observed from HYPR-OSEM likely due to the difference in resolution between the composite and the target image. In general, HYPR does not degrade the resolution when incorporated directly within OSEM.

In figure $\mathrm{S} 2$, the CRC yalues are plotted against image noise for various sizes of hot and cold regions obtained from various reconstruction algorithms. It was observed that the convergence in CRC for all forms of HYPR-OSEM was slower than that of OSEM; e.g. the CRC obtained from 3 iterations of OSEM was similar to that obtained from 9 iterations of HYPR-AU-OSEM. Nevertheless, HYPR successfully reduced the noise increment per iteration while allowing effective update of contrast. Specifically, the image noise was 2-3 times lower with HYPR-OSEM for an equivalent CRC value, and higher CRC values were 
observed at the same noise level as compared to OSEM. Note that slightly higher CRC's obtained from HYPR-OSEM as compared to OSEM after convergence could be due to the slightly better resolution performance and lower noise-induced bias (i.e. the maximum likelihood estimate is only unbiased for an infinite number of counts). When comparing to the filtered OSEM, one can observe that HYPR-OSEM images contain similar noise levels but with higher CRC due to the preservation of resolution in the HYPR-OSEM images. As expected, the post reconstruction filter reduced the noise at the cost of (reduced) resolution and contrast for the filtered OSEM, and the reduction of CRC's due to the post filter is greater for smaller hot structures. In general, HYPR-OSEM can achieve better accuracy at equivalent noise level as well as better precision than OSEM and better accuracy than filtered OSEM.

When comparing among the different forms of HYPR-OSEM, it was observed that HYPR-AU-OSEM has a faster convergence in CRC than the other two forms especially for the cold regions and the smallest $(10 \mathrm{~mm})$ hot region. In addition, the level of noise reduction was quite similar among the different forms of HYPR-OSEM when the filter kernel used in the HYPR operator was $5 \mathrm{~mm}$ FWHM, which is similar to the intrinsic resolution of the simulated PET system. The kernel which achieves similar noise reduction across all HYPROSEM methods will be referred to as the Noise Equivalent Kernel (NEK) from here on. The effects of different filter kernel sizes will be presented shortly. The reference and reconstructed phantom images are depicted in figure S3. One can observe visually that HYPR-AU-OSEM image contains higher CRC particularly in the cold regions but also slightly higher noise than HYPR-F-OSEM and HYPR-FB-OSEM images for the same number of iterations in this case. 


\section{Evaluation of effect of filter kernel size}

In terms of noise reduction, the wider the filter kernel the higher the noise reduction. Furthermore, the widest kernel produced the slowest convergence in CRC especially in the cold regions as well as for the smallest hot region. However, the noise increment per iteration was not very different when using different kernel sizes in the HYPR operator of HYPR-FOSEM. Very similar results were obtained from HYPR-FB-OSEM. In other words, the noise reduction achieved by HYPR-F-OSEM and HYPR-FB-OSEM is not very dependent on the kernel size. In contrast, 3 distinct CRC vs noise trajectories were observed from the 3 different kernel sizes for HYPR-AU-OSEM as shown in figure S4. One can easily observe that the narrowest kernel (red) achieved the least noise reduction (i.e. noise level was $\sim 2$ times lower than that of OSEM) with the fastest convergence in CRC among the 3 different kernel sizes, and the number of iterations required to reach a good CRC was comparable to that of OSEM. The widest kernel (green) achieved the highest noise reduction (much more than what was achieved by HYPR-F-OSEM and HYPR-FB-OSEM); however, it also produced the slowest convergence in the cold regions as well as for the smallest hot region. Up to $\sim 4$ times lower noise level than that of OSEM can be achieved by HYPR-AU-OSEM using the $10 \mathrm{~mm}$ filter kernel in this case. Note that the 'perfect' CRC vs noise trajectory would be a straight vertical line (i.e. improving CRC without increasing noise).

The reconstructed contrast phantom images with different filter kernel sizes used in the HYPR operator are depicted in figure S5 for all forms of HYPR-OSEM. The effect of different kernel sizes is not very apparent visually for both HYPR-F-OSEM and HYPR-FBOSEM, while the visual difference among the HYPR-AU-OSEM images with different kernel sizes can be easily observed. When the kernel used in the HYPR operator is narrower than the NEK, HYPR-AU-OSEM performs worse than both HYPR-F-OSEM and HYPR-FBOSEM in terms of noise reduction. More high frequency noise or 'false objects' can be 
observed in the HYPR-AU-OSEM image (see figure S5g). Conversely, when the kernel is wider than the NEK, HYPR-AU-OSEM reduces the high frequency noise to a larger degree than the other two forms, although at the expense of a slower convergence (see figure S5i). In addition, similar resolution performance was observed for different kernel sizes for all forms of HYPR-OSEM (not shown).

\section{Precision, accuracy, and root mean squared error}

The plots of \%COV vs \%Bias (i.e. precision vs accuracy) in CRC generated from multiple realizations of the contrast phantom for various reconstruction methods are depicted in figure S6. In general, all forms of HYPR-OSEM achieved lower \%COV or better precision than both OSEM and filtered OSEM. As shown in figure S6c, the highest improvement was observed for the $15 \mathrm{~mm}$ hot region where a $20 \%$ improvement in accuracy (i.e. 20\% lower bias) for a fixed $20 \% \mathrm{COV}$ and a $\sim 5 \%$ lower COV for a fixed $\sim 1 \%$ bias was achieved by HYPR-AU-OSEM as compared to OSEM. When comparing to filtered OSEM, a 15\% improvement in accuracy for a fixed $20 \% \mathrm{COV}$ and a $\sim 5 \%$ lower COV for a fixed $\sim 12 \%$ bias was achieved by HYPR-AU-OSEM. Note that the post filter used to generate filtered OSEM images only degraded the accuracy in CRC without improving the precision at all for the largest hot region and the cold regions (see figures S6a, e, and f). It is also very apparent that the HYPR-AU-OSEM has faster convergence than the other two forms of HYPR-OSEM when NEK was used as observed previously. In addition, for the smallest hot region the $\% \mathrm{COV}$ vs \%Bias curves for all reconstructions followed similar trajectory except for the filtered OSEM at high number of iterations. This is most likely due to the size of the region which is very close to the resolution limit of the simulated PET system (i.e. only 2 times the system resolution). 
Figure $\mathrm{S} 7$ shows the \%RMSE in CRC as a function of number of iterations for various reconstruction methods. For the hot regions, OSEM and filtered OSEM achieved their optimal performance at 2-3 iterations which correspond to the number of iterations used in typical clinical protocols. More iterations were required for all HYPR-OSEM methods to reach the lowest \%RMSE as compared to OSEM for both hot and cold regions. One interesting observation is that at the 2nd iteration where the HYPR operator was first introduced into the HYPR-OSEM methods, the \%RMSE increased for HYPR-F-OSEM and HYPR-FB-OSEM as compared to that from the initial OSEM iteration, whereas that obtained from HYPR-AU-OSEM decreased at the 2nd iteration. As a result, much more iterations were needed for HYPR-F-OSEM and HYPR-FB-OSEM to reach similar \%RMSE as compared to HYPR-AU-OSEM especially for the cold regions and small hot regions. Additionally, HYPR-OSEM is expected to outperform OSEM and filtered OSEM in terms of \%RMSE particularly for small regions since it achieves better precision and similar accuracy as compared to OSEM, and better accuracy and similar precision as compared to filtered OSEM. However, the highest improvement was not observed from the smallest hot region; this is likely due to that the size of the region is near the resolution limit of the PET system.

\section{Experimental contrast phantom scan}

The CRC vs noise for the high count frame of the experimental contrast phantom data is shown in figure 2. The noise reduction achieved by HYPR-OSEM with respect to OSEM for the HRRT data was higher than that for the simulated data. Nearly identical results were obtained between HYPR-F-OSEM and HYPR-FB-OSEM. The noise level obtained from HYPR-F-OSEM and HYPR-FB-OSEM was 3-4 times lower than that from OSEM, while that obtained from HYPR-AU-OSEM was $\sim 6$ times lower. The different noise reduction between HYPR-F(B)-OSEM and HYPR-AU-OSEM is likely due to the fact that the $2.5 \mathrm{~mm}$ kernel used in the HYPR operator does not correspond to the NEK. Consequently, the NEK 
does not always correspond to the intrinsic resolution of the PET system, and the NEK for the HRRT data is thus narrower than $2.5 \mathrm{~mm}$ FWHM.
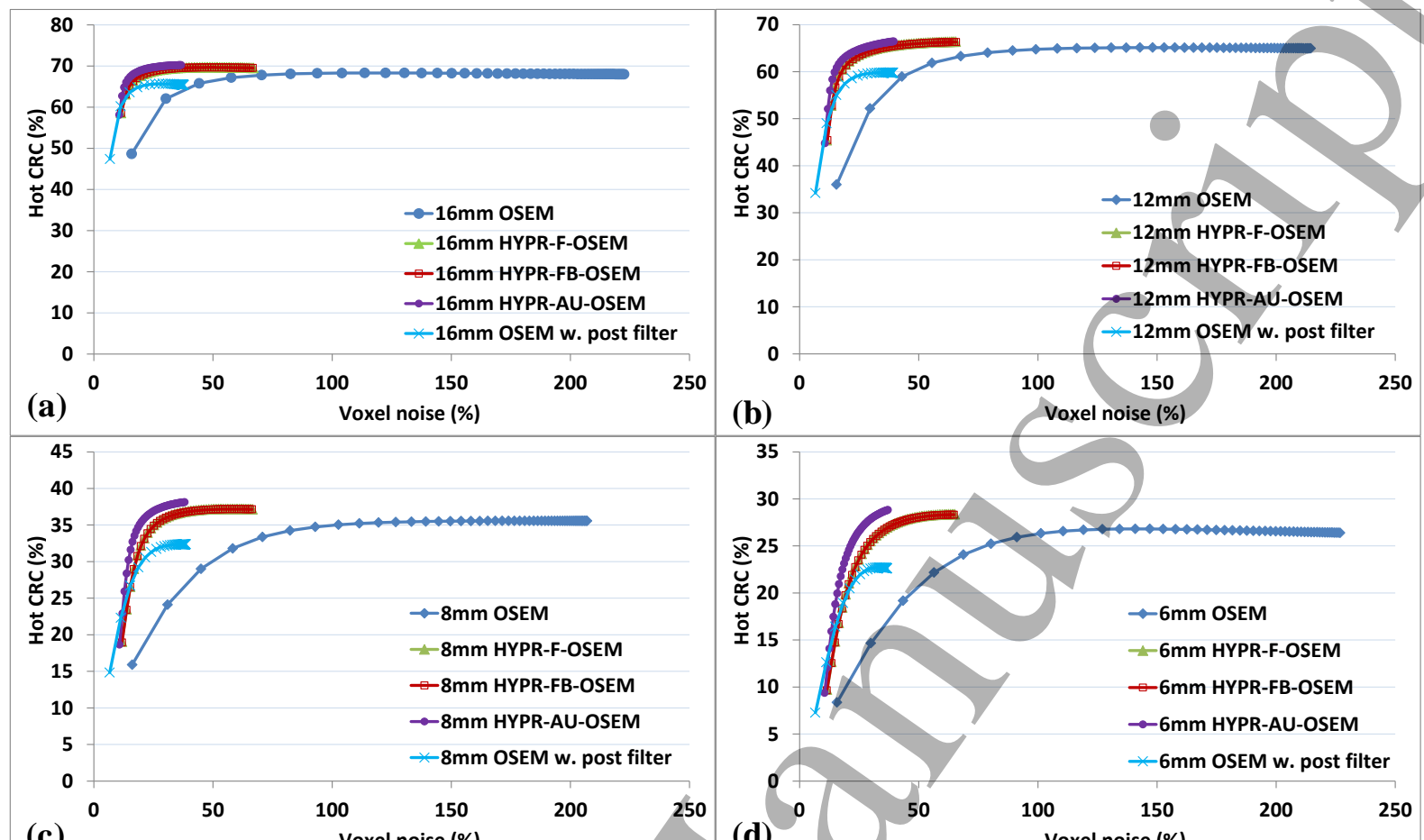

(c)

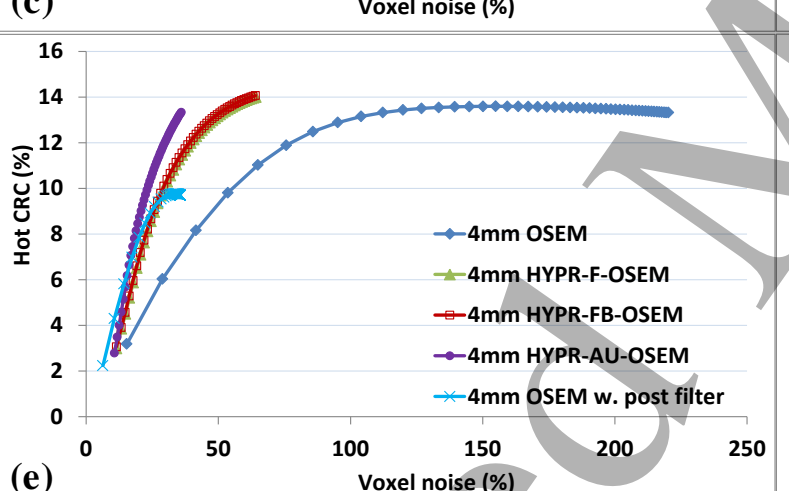

(b) Voxel noise (\%)

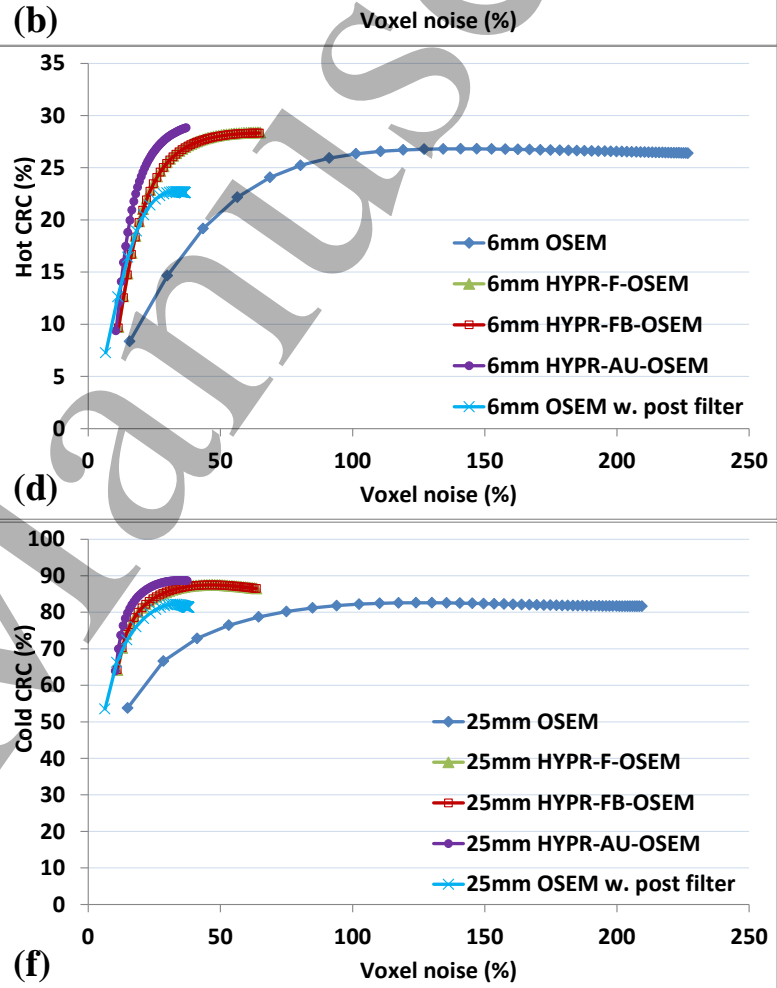

Figure 2. CRC against voxel-noise for various sizes of hot regions: (a) $16 \mathrm{~mm}$, (b) $12 \mathrm{~mm}$, (c) $8 \mathrm{~mm}$, (d) 6 $\mathrm{mm}$, (e) $4 \mathrm{~mm}$ in diameter, and (f) for the $25 \mathrm{~mm}$ diameter cold region obtained from the high count frame of the experimental contrast phantom data reconstructed by OSEM, HYPR-F-OSEM, HYPR-FBOSEM, HYPR-AU-OSEM, and OSEM with a post reconstruction filter. 


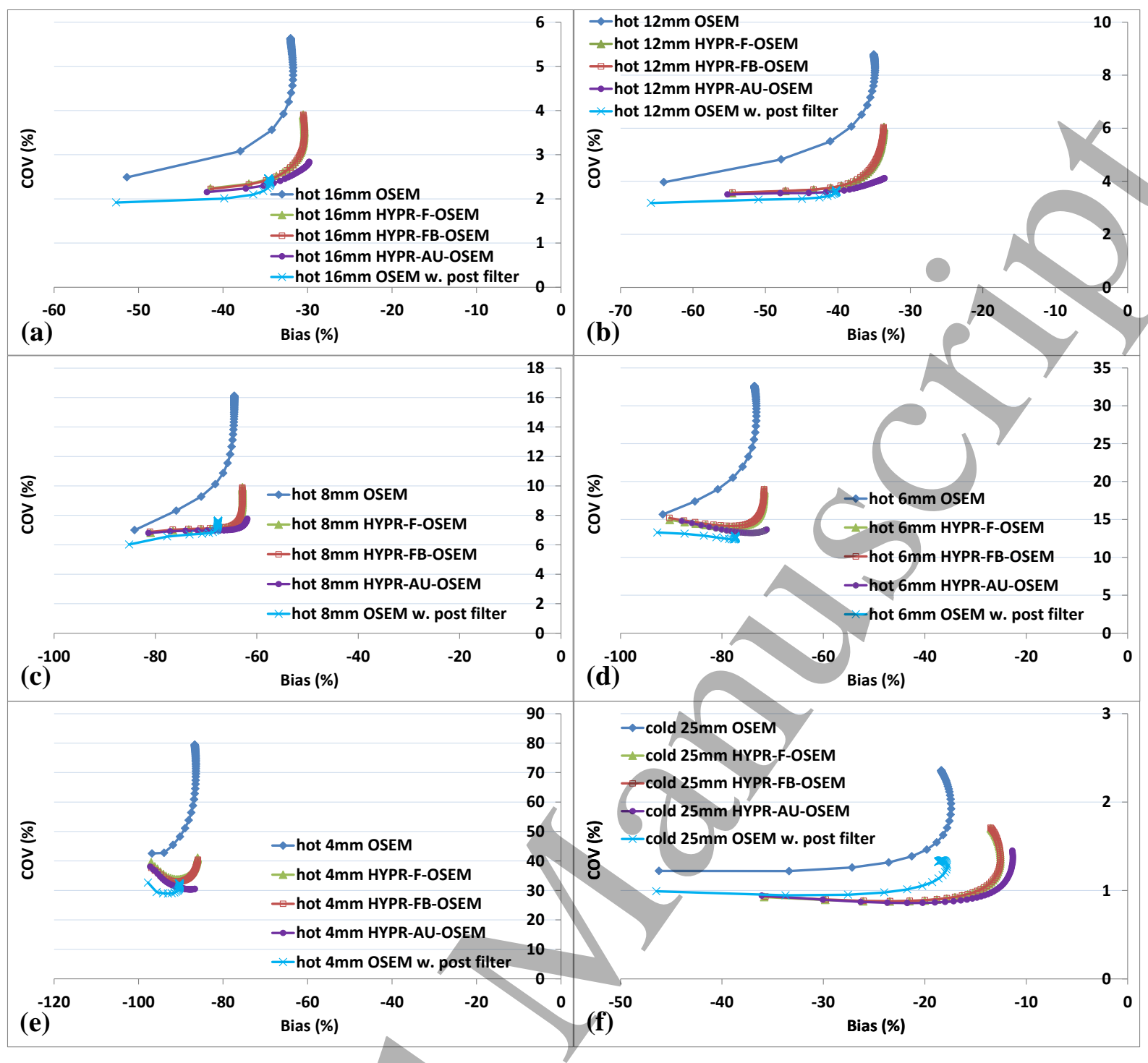

Figure 3. \% COV against \% Bias of CRC values for various sizes of hot regions: (a) $16 \mathrm{~mm}$, (b) $12 \mathrm{~mm}$, (c) $8 \mathrm{~mm}$, (d) $6 \mathrm{~mm}$, (e) $4 \mathrm{~mm}$ in diameter, and (f) for the $25 \mathrm{~mm}$ diameter cold region obtained from the high count frame of the experimental phantom data reconstructed by OSEM, HYPR-F-OSEM, HYPR-FBOSEM, HYPR-AU-OSEM, and OSEM with post reconstruction filter.

The $\% \mathrm{COV}$ vs $\%$ Bias obtained from 50 realizations of the high count experimental phantom data is depicted in figure 3. As expected, HYPR-OSEM achieved better precision than OSEM and better accuracy than filtered OSEM. As compared to the simulation results from figure S6, HYPR-AU-OSEM did not show faster convergence than HYPR-F-OSEM and HYPR-FB-OSEM in this case. This could be due to that the filter kernel used in the HYPR operator is wider than the NEK (i.e. convergence of HYPR-AU-OSEM became slower). On the other hand, HYPR-AU-OSEM achieved better precision in CRC for all hot and cold regions than HYPR-F-OSEM and HYPR-FB-OSEM. Here the highest improvement 
in \%COV achieved by HYPR-OSEM was observed for the $4 \mathrm{~mm}$ hot insert; e.g. 30\% improvement in precision was achieved by HYPR-AU-OSEM as compared to OSEM at an equivalent $\%$ Bias level.

Similar accuracy in hot CRC's was obtained from all methods except for the filtered OSEM which showed highest \%Bias in all cases. As for the cold CRC, a 7\% improvement was observed from HYPR-AU-OSEM as compared to OSEM, likely as a result of the more effective update of cold contrast with a very low noise increment per update (i.e. lower noiseinduced bias). Note that much higher \%Bias was obtained from the experimental phantom data as compared to the simulations. This is most likely due to the fact that the reference CRC's for simulations are known from the noise-free case, and the partial volume effect was included in the reference CRC's, whereas the reference CRC's are unknown for the experimental phantom data, and $100 \%$ was used as the reference CRC for all regions.

Figure 4 shows the \%RMSE in CRC obtained from the high count frame of the experimental phantom data. As depicted in figure 3, the \%Bias was much higher than the $\% \mathrm{COV}$ in this case. As a result, bias contributed more to the \% RMSE than the variance. Consequently, \%RMSE for OSEM was always lower than that for the filtered OSEM in spite of a higher \%COV. As expected, HYPR-OSEM methods attained lower \%RMSE than OSEM and filtered OSEM in all cases after certain numbers of iterations (more iterations are need for smaller regions). All HYPR-OSEM methods performed similarly in terms of \%RMSE in this case. 

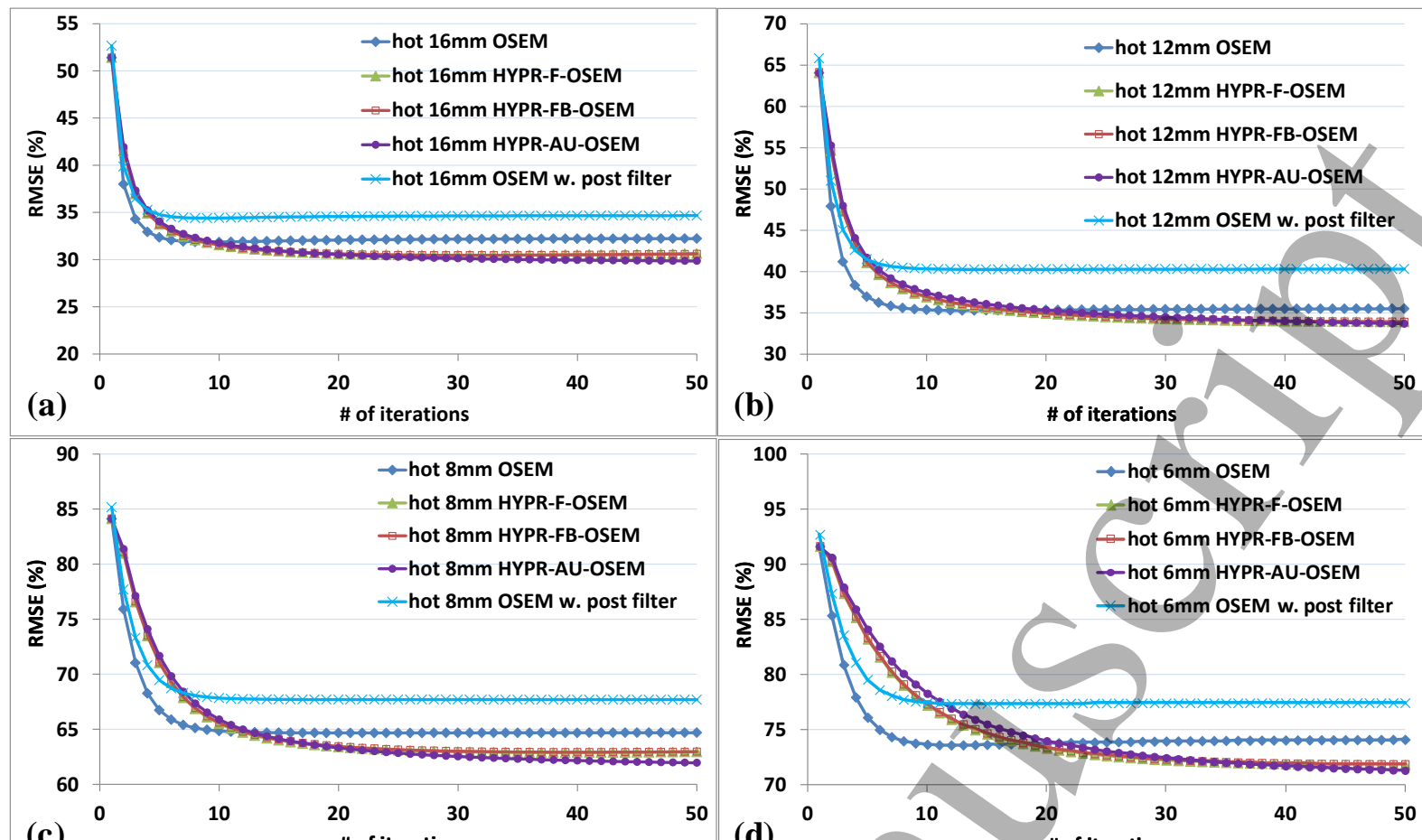

(b) $\quad \begin{array}{lll}0 & & \\ & \text { \# of iterations }\end{array}$
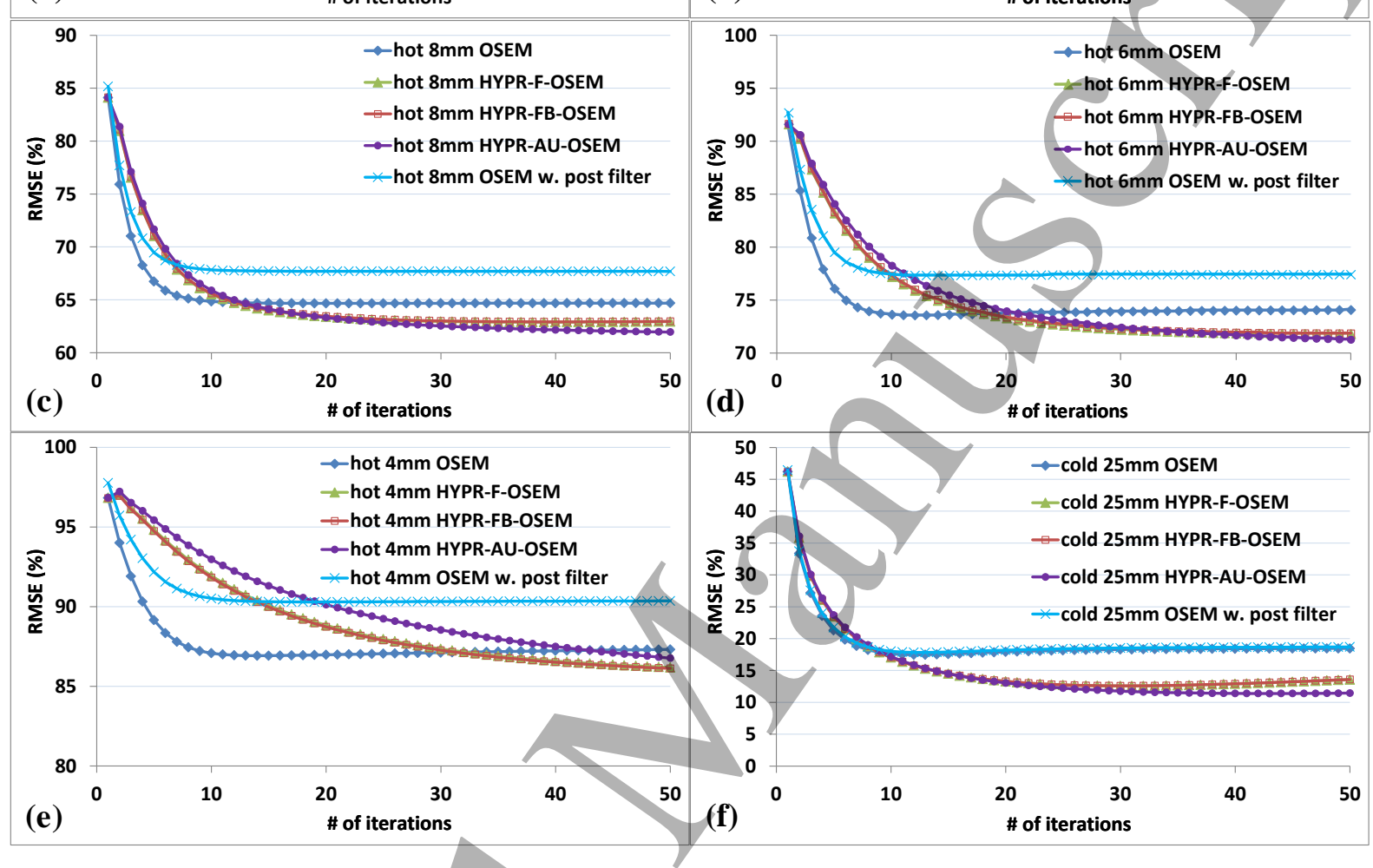

Figure 4. \% RMSE in CRC as a function of number of OSEM iterations for various sizes of hot regions: (a) $16 \mathrm{~mm}$, (b) $12 \mathrm{~mm}$, (c) $8 \mathrm{~mm}$, (d) $6 \mathrm{~mm}$, (e) $4 \mathrm{~mm}$ in diameter, and (f) for the $25 \mathrm{~mm}$ diameter cold region obtained from the high count frame of the experimental phantom data reconstructed by OSEM, HYPR-F-OSEM, HYPR-FB-OSEM, HYPR-AU-OSEM, and OSEM with post reconstruction filter.

Similar comparisons for the low count frame of the experimental phantom data are depicted in figures 5-7. In contrast to the results from the high count data, the noise level obtained from HYPR-AU-OSEM was not in agreement with that from the filtered OSEM anymore. This is due to the fact that the composite is noisier than the filtered target in this case. Therefore, the noise in the de-noised image is dominated by the noise in the composite for the low count case, while the noise in the de-noised image is dominated by the noise in the filtered target for the high count case. Furthermore, higher noise-induced bias in CRC was observed from OSEM since there were not enough counts within each OSEM subset to 
produce accurate CRC's. Consequently, improvements in both accuracy and precision were achieved by HYPR-OSEM as compared to OSEM in all cases except for the smallest hot region. Similar CRC for the smallest hot region between HYPR-OSEM and OSEM methods is likely due to the size of the region being close to the resolution limit of the HRRT.
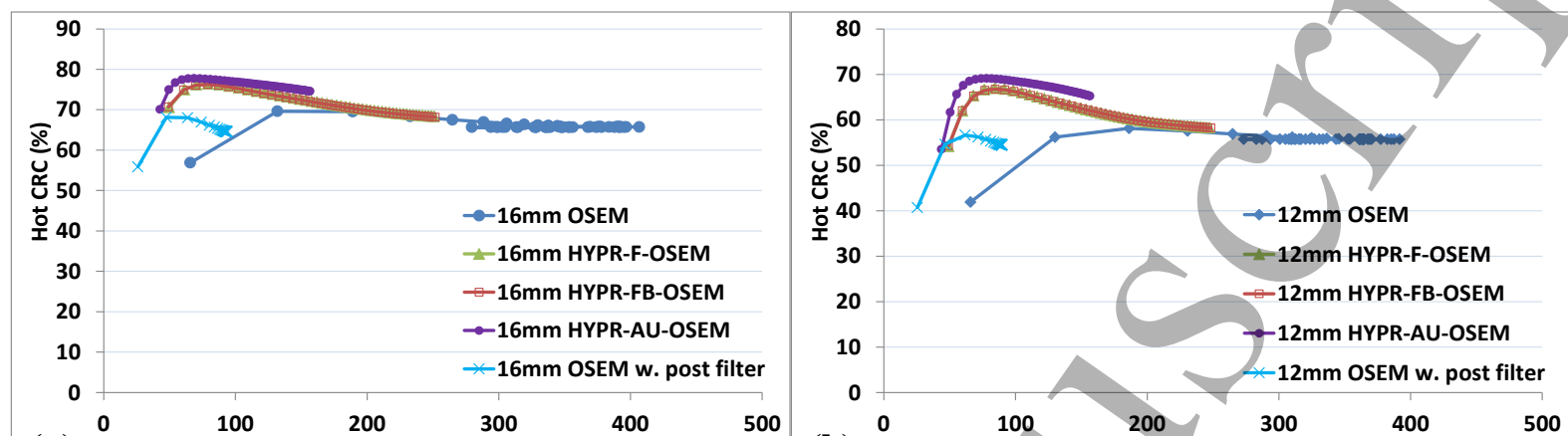

(a) Voxel noise (\%) (b)

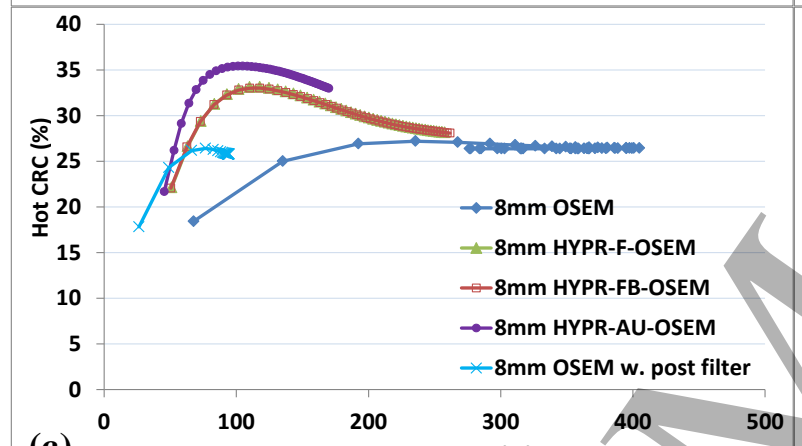

(c) Voxel noise (\%)
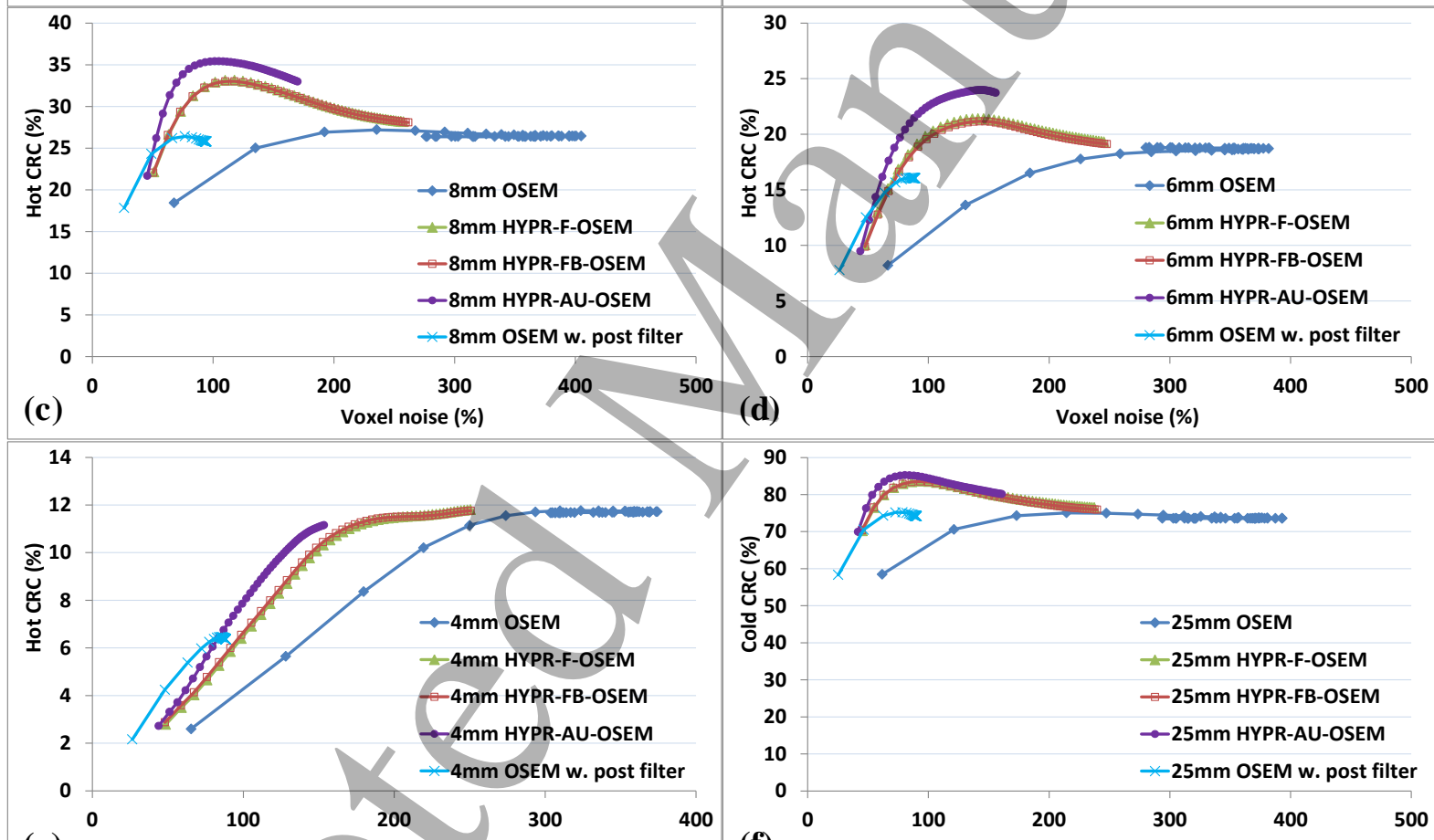

(e)

Voxel noise (\%)

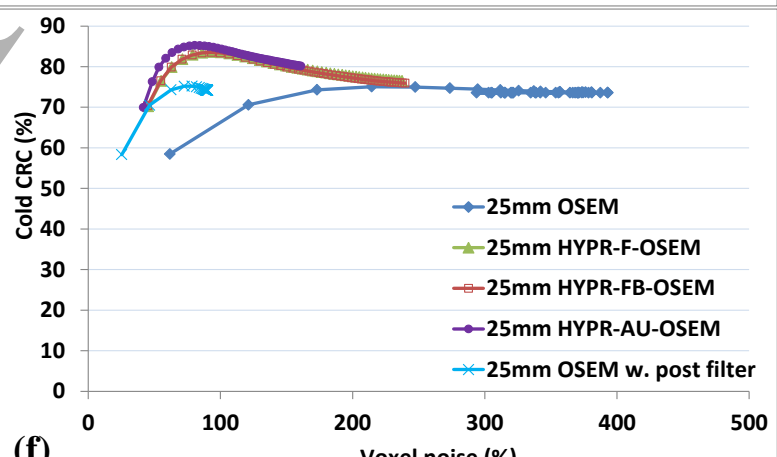

Figure 5. CRC against voxel-noise for various sizes of hot regions: (a) $16 \mathrm{~mm}$, (b) $12 \mathrm{~mm}$, (c) $8 \mathrm{~mm}$, (d) 6 $\mathrm{mm}$, (e) $4 \mathrm{~mm}$ in diameter, and (f) for the $25 \mathrm{~mm}$ diameter cold region obtained from the low count frame of the experimental contrast phantom data reconstructed by OSEM, HYPR-F-OSEM, HYPR-FB-OSEM, HYPR-AU-OSEM, and OSEM with a post reconstruction filter. 


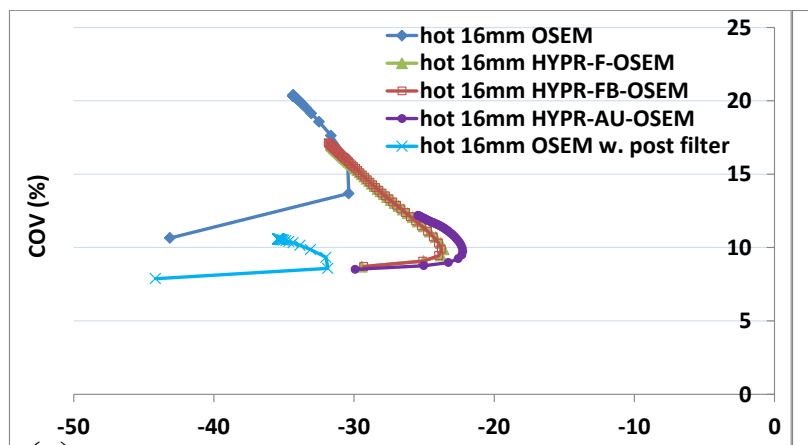

(a) $\quad-40 \quad-10$ Bias (\%) (b) Bias (\%)
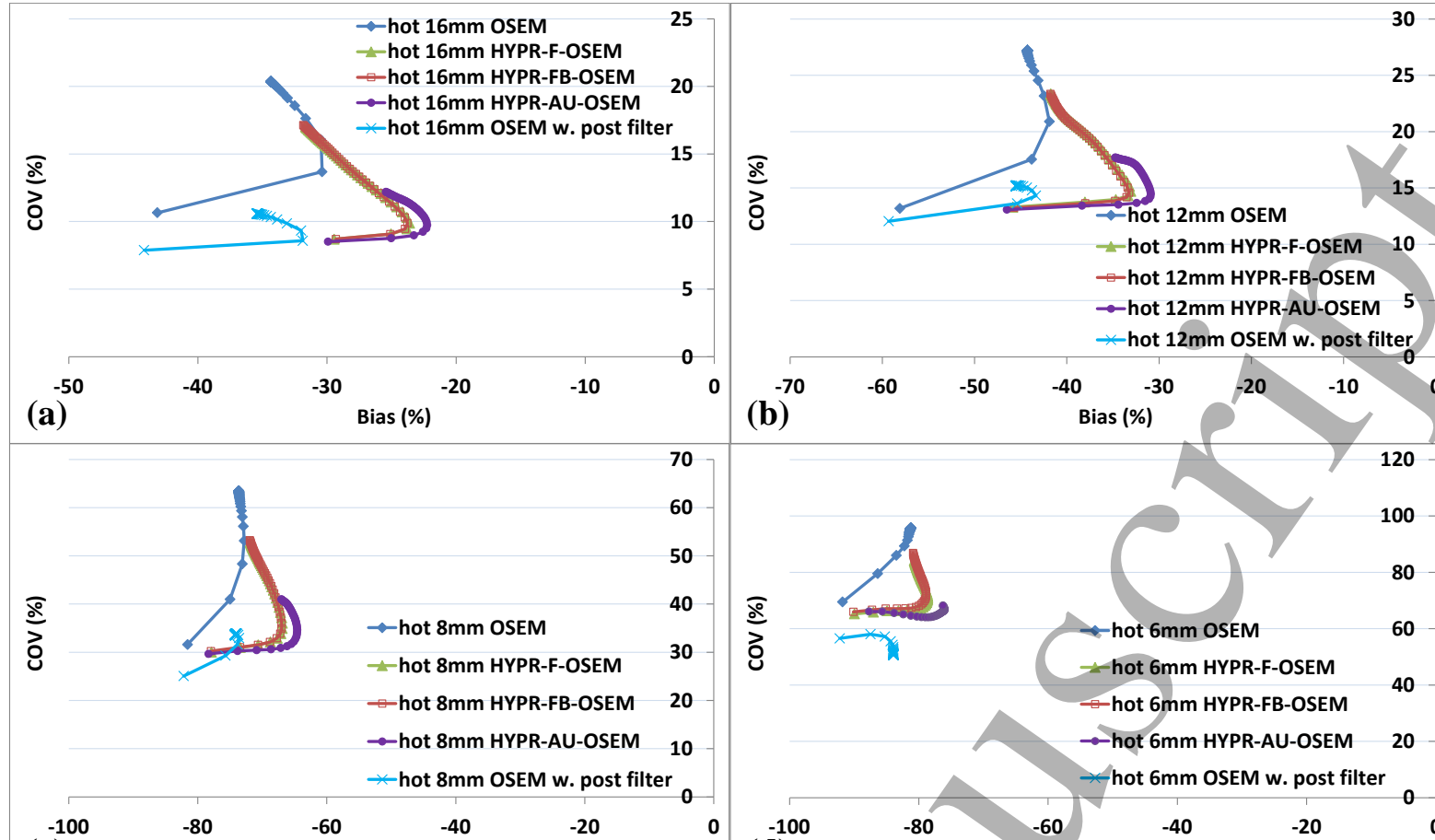

\begin{tabular}{lllll}
$($ (c) & -80 & -60 & -20 & 0 \\
& & Bias (\%) & & \\
\hline
\end{tabular}

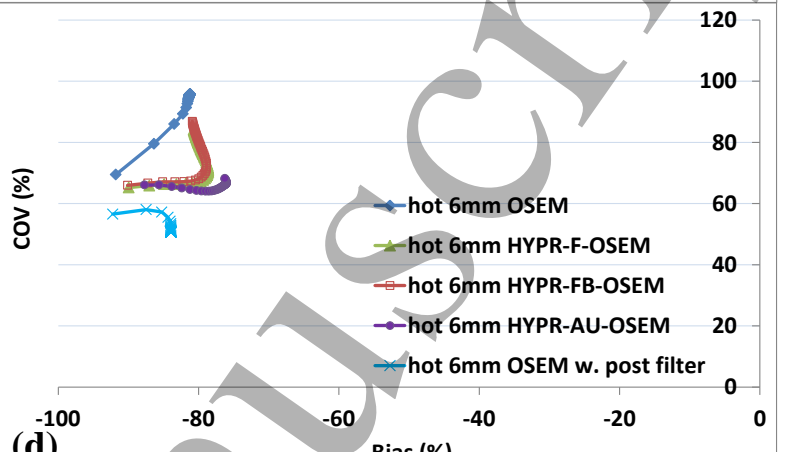

(d) Bias (\%)

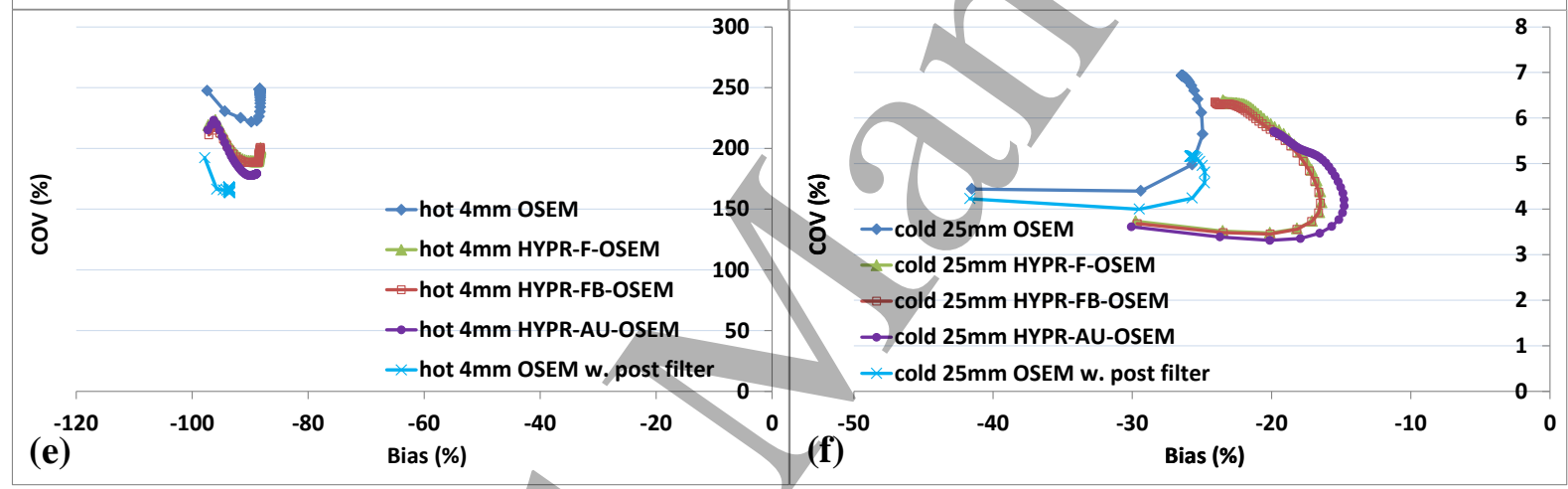

Figure 6. \% COV against \% Bias of CRC values for various sizes of hot regions: (a) $16 \mathrm{~mm}$, (b) $12 \mathrm{~mm}$, (c) $8 \mathrm{~mm}$, (d) $6 \mathrm{~mm}$, (e) $4 \mathrm{~mm}$ in diameter, and (f) for the $25 \mathrm{~mm}$ diameter cold region obtained from the low count frame of the experimental phantom data reconstructed by OSEM, HYPR-F-OSEM, HYPR-FBOSEM, HYPR-AU-OSEM, and OSEM with post reconstruction filter.

With regard to \%COV vs \% Bias at low count level, the noise-induced bias was very apparent for many cases; i.e. the \%Bias became worse when the iterations passed a certain number. Moreover, higher noise-induced bias was observed from HYPR-F-OSEM and HYPR-FB-OSEM as compared to HYPR-AU-OSEM. As a result, substantial difference in \%RMSE can now be observed between HYPR-F(B)-OSEM and HYPR-AU-OSEM as shown in figure 7. In terms of \%RMSE, OSEM and filtered OSEM performed similarly in this case. Greater improvement in \%RMSE with respect to OSEM was attained by HYPR-AU-OSEM

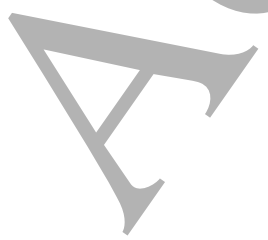


as compared to the high count case due to the higher noise-induced bias in OSEM at low count level.
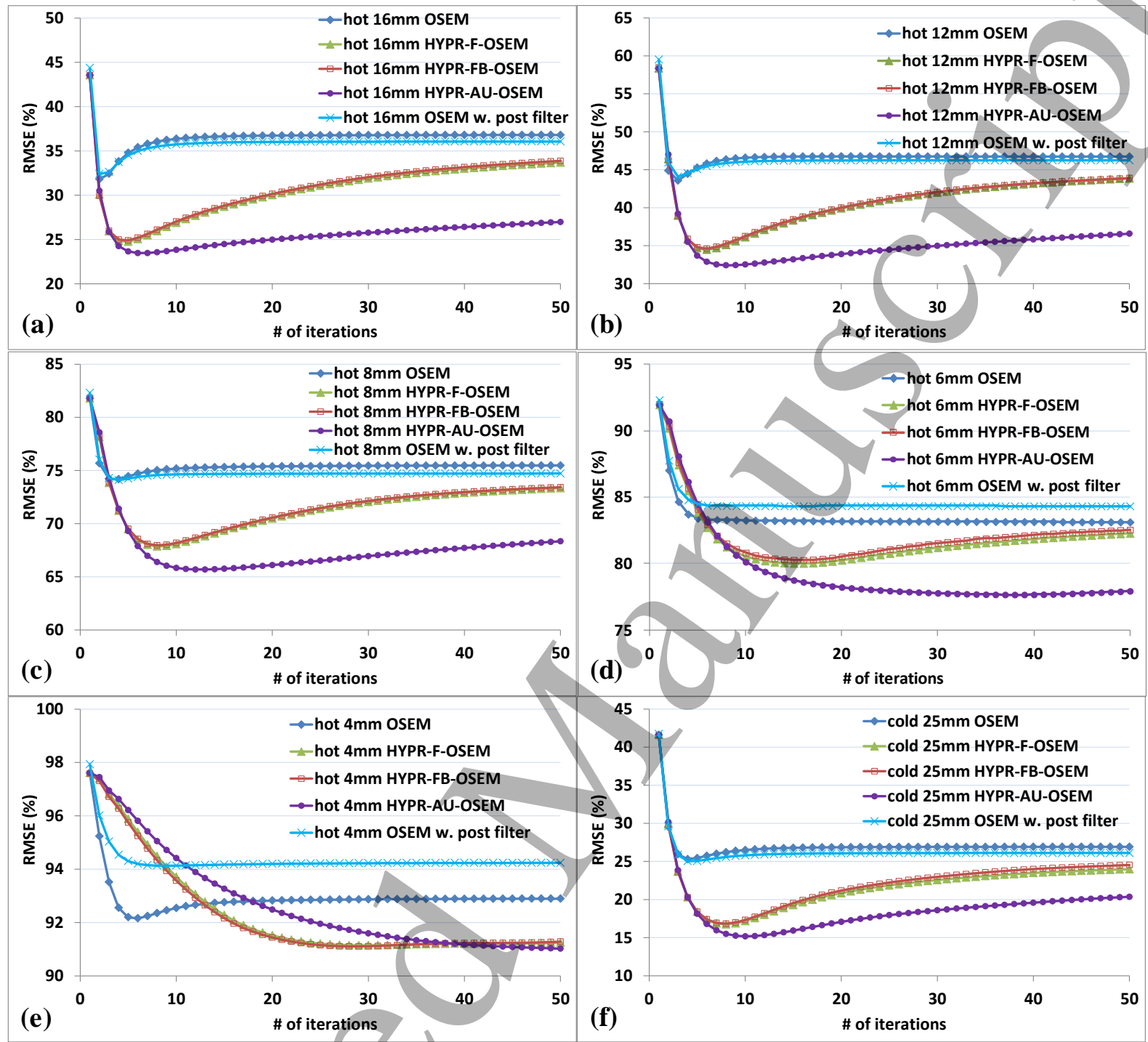

Figure 7. \% RMSE in CRC âs a function of number of OSEM iterations for various sizes of hot regions: (a) $16 \mathrm{~mm}$, (b) $12 \mathrm{~mm}$, (c) $8 \mathrm{~mm}$, (d) $6 \mathrm{~mm}$, (e) $4 \mathrm{~mm}$ in diameter, and (f) for the $25 \mathrm{~mm}$ diameter cold region obtained from the low count frame of the experimental phantom data reconstructed by OSEM, HYPR-F-OSEM, HYPR-FB-OSEM, HYPR-AU-OSEM, and OSEM with post reconstruction filter. 

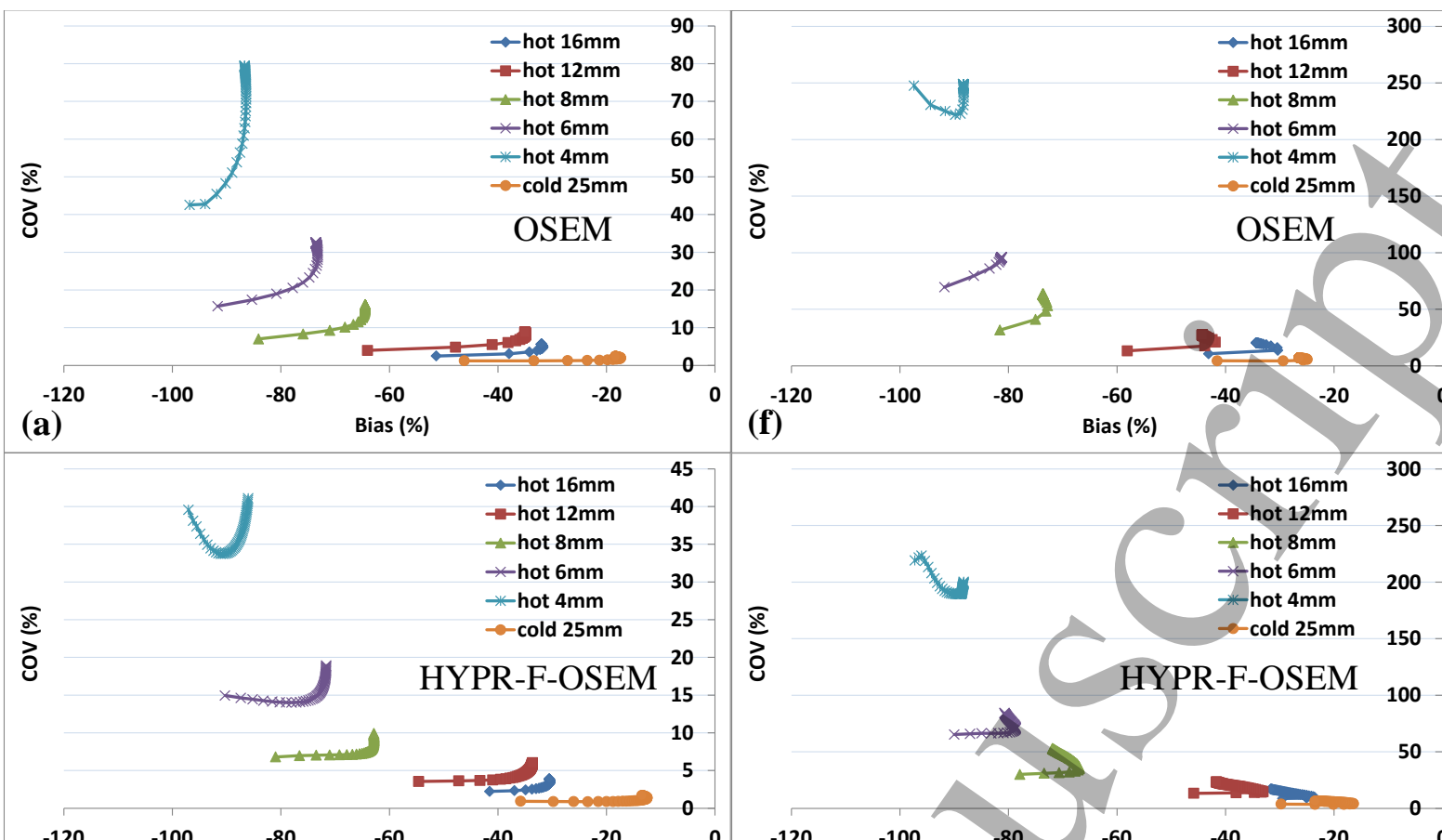

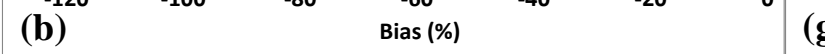

(f) Bias (\%)

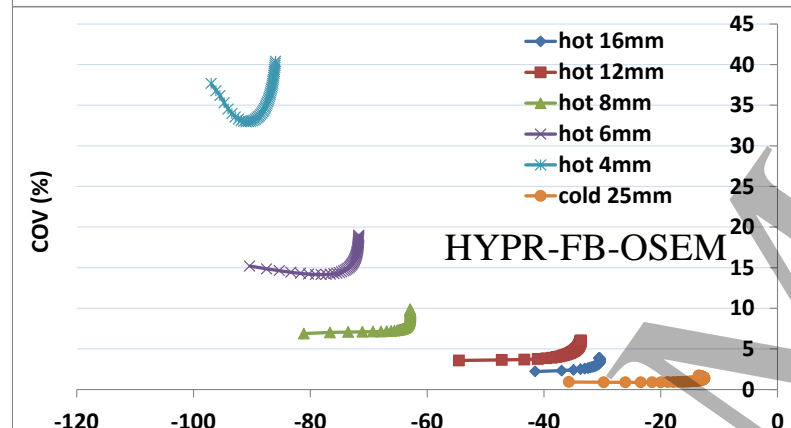

(g) Bias (\%)

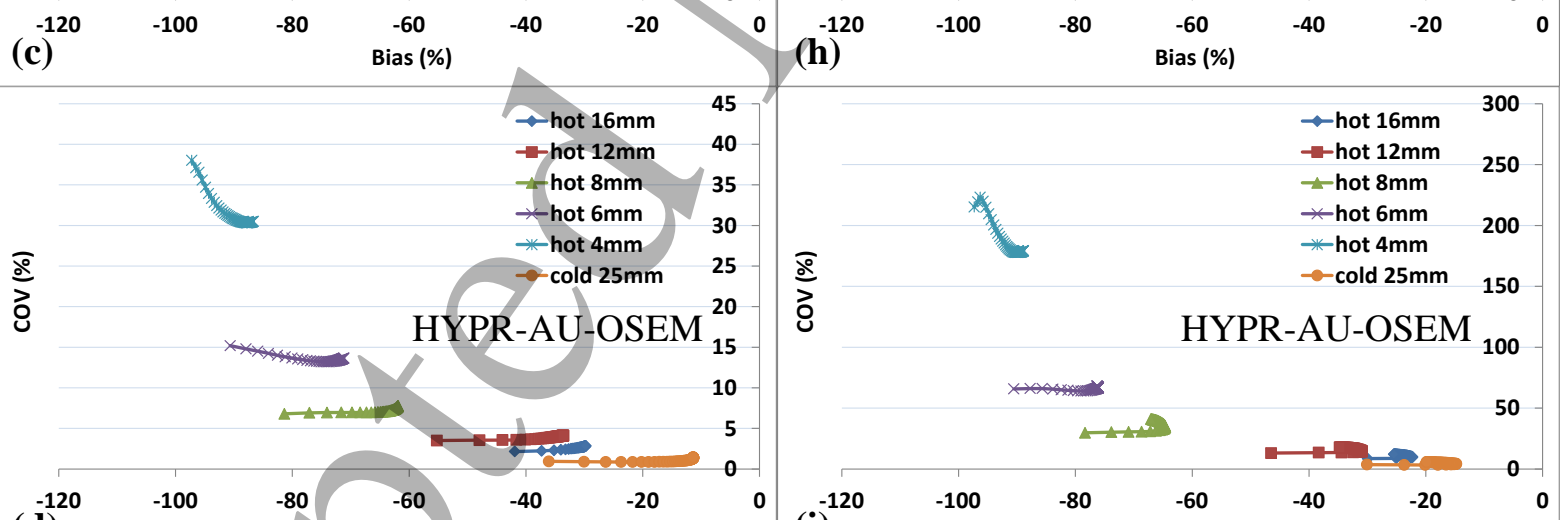

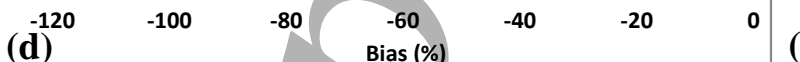

$\begin{array}{lllc}\text { (i) }^{-120} & -100 & -80 & -60 \\ & & & \text { Bias (\%) }\end{array}$
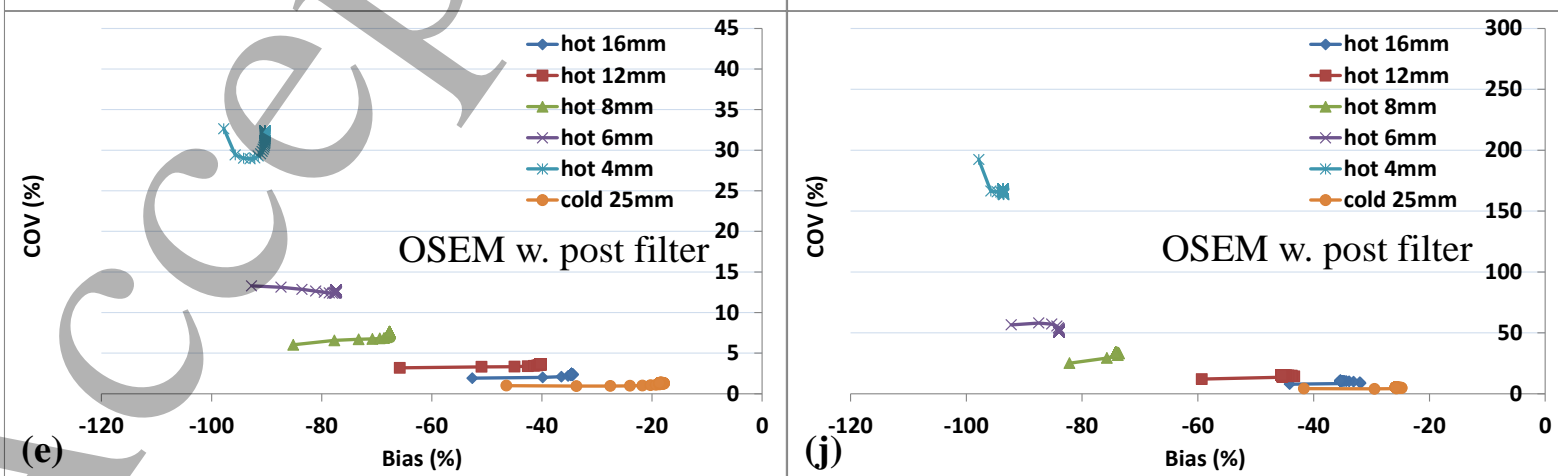
Figure 8. \% COV against \% Bias of CRC values for various sizes of hot and cold regions obtained from (a) OSEM, (b) HYPR-F-OSEM, (c) HYPR-FB-OSEM, (d) HYPR-AU-OSEM, and (e) OSEM with post reconstruction filter (i.e. filtered OSEM) at high count level. (f) - (j) show the same analyses at low count level. Note that the maximum of the COV axis in panel (a) is higher than that in (b) - (e) since OSEM has the highest $\mathrm{COV}$ or the worst reproducibility in general.

Figure 8 summarizes all the $\% \mathrm{COV}$ vs $\%$ Bias curves obtained from various sizes of hot and cold regions for each reconstruction method at high (figure 8a-e) and low count (figure $8 \mathrm{f}-\mathrm{j}$ ) levels. One can easily observe that OSEM is the worst in terms of precision/reproducibility of CRC values (i.e. highly dependent on the number of OSEM iterations), while HYPR-AU-OSEM shows the most stable performance in terms of reproducibility as the number of iterations increases. Another interesting observation is that the curves obtained from the filtered OSEM follow similar trajectories as those obtained from HYPR-AU-OSEM since they achieve similar precision. However, those obtained from the filtered OSEM appear as the 'truncated' versions of those obtained from the HYPR-AUOSEM; i.e. the accuracy does not improve anymore after a certain number of iterations due to the loss of resolution in the filtered OSEM images.

\section{Clinical patient study}

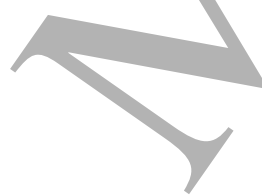

Evaluation using the PK11195 patient scan is depicted in figure 9. One can observe that HYPR-AU-OSEM images are less noisy than OSEM images, while HYPR-AU-OSEM images show higher contrast in both hot and cold regions as compared to the filtered OSEM. Additionally, cold regions are also more visible in the HYPR-AU-OSEM image; e.g. cold eyes and cold tumor as indicated by the red arrows in figure 9e and $\mathrm{f}$. Although there is no gold-standard for the case of the patient study, these observations agree with those observed previously from simulations and experimental phantom studies. 

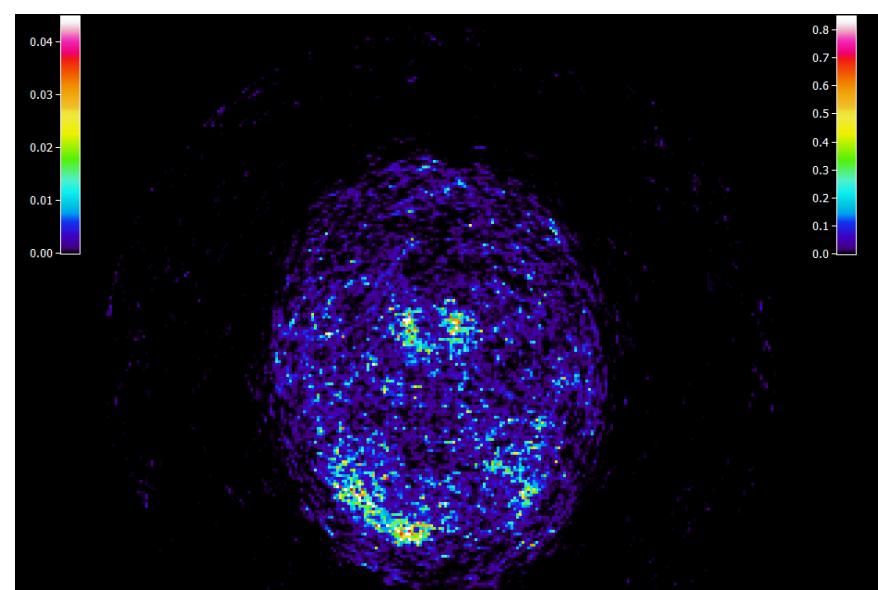

(a)

(d)
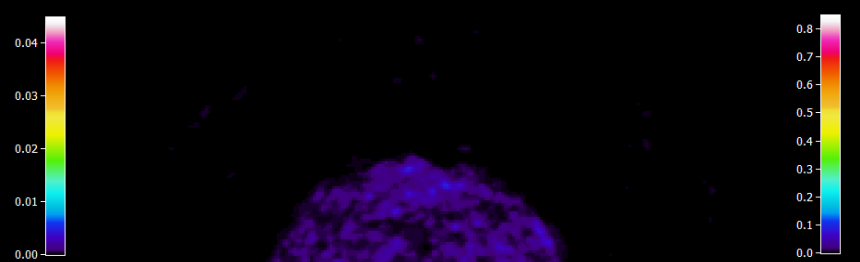

(b)

(e)
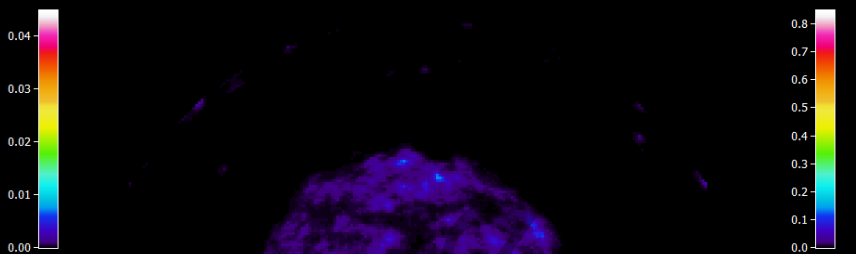

(c)

Figure 9. PK11195 patient data reconstructed with (a) OSEM, (b) OSEM with a post reconstruction filter, and (c) HYPR-AU-OSEM for the 1st dynamic frame (low count). (d) - (f) show the corresponding reconstructed images (different slice) for the 9th dynamic frame (high count). The number of iterations which achieved the lowest \%RMSE according to the phantom experiment was used for each reconstruction. The same color scale was used across each column of the images. The arrows highlight the 
internal carotid arteries and transverse sinus (blood vessels) in (b) and (c) while they indicate the eyes and tumour in (e) and (f).

\section{Discussions}

From the contrast phantom simulations and experimental phantom data at high count level, it was observed that HYPR-OSEM images have similar CRC as compared to OSEM but with the noise level comparable to that from the filtered OSEM (with a filter kernel size matching with the one used in the HYPR operator). In other words, HYPR-OSEM images contain good traits from both OSEM and the filtered OSEM images. It was later observed from the experimental phantom study at low count level that HYPR-OSEM images contain lower noise-induced bias in CRC than both OSEM and filtered OSEM images. However, higher noise was observed in the HYPR-OSEM images as compared to the filtered OSEM due to the higher noise in the composite as previously described.

When comparing among different forms of HYPR-OSEM, it was observed that the convergence rate in CRC for HYPR-AU-OSEM was faster than that for HYPR-F-OSEM and HYPR-FB-OSEM when a kernel size equivalent to or narrower than that of NEK was used in the HYPR operator based on the simulations. Furthermore, it was observed that the CRC vs noise trajectories obtained from HYPR-F-OSEM and HYPR-FB-OSEM were not sensitive to the kernel size used in the HYPR operator. On the other hand, higher noise reduction and better CRC vs noise trajectories can be achieved by HYPR-AU-OSEM using wider kernels, though the convergence of HYPR-AU-OSEM becomes slower. Moreover, it was found that the NEK does not always correspond to the intrinsic resolution of the PET system from the experimental phantom study. When a kernel size wider than that of NEK was used for the phantom experiment, higher noise reduction was achieved by HYPR-AU-OSEM as compared to the other two forms as expected. In addition, lower noise-induced bias at low count level and higher reproducibility was generally observed for HYPR-AU-OSEM. 
Consequently, based on the results obtained from this work, separating the HYPR operator from the computation of the update factors seems to be more effective in terms of accuracy and precision performance than introducing HYPR directly within the computation of update factors.

As mentioned previously, the HYPR operator was incorporated within OSEM after only one iteration of standard OSEM in order to achieve high SNR gain and ayoid high frequency noise in the composite. Although running more iterations of standard OSEM initially can improve the convergence in CRC's and minimize the small variation in resolution, the absolute noise level at later iterations of OSEM is much higher than that at the first iteration especially under low count situations. Therefore, it is hard to justify the starting point for incorporating the HYPR operator after more than one iteration of OSEM. As shown in figure $\mathrm{S} 2$, one can observe that the noise level at the $2^{\text {nd }}$ iteration of OSEM was even higher than that at $16^{\text {th }}$ iteration of all forms of HYPR-OSEM. Similar trends can also be observed from the experimental phantom results (figures 2 and 5). Using the composite derived from the current iteration of subset images can also improve the convergence of HYPR-OSEM. However, this would result in lower noise reduction as compared to using the composite from the previous iteration; i.e. the CRC vs noise trajectory became very close to that of OSEM (results not shown).

Most of the results in this work were obtained using the $5 \mathrm{~mm}$ FWHM Gaussian kernel in the HYPR operator for the simulations and $2.5 \mathrm{~mm}$ FWHM for the HRRT data as a compromise between noise reduction and convergence since a very narrow kernel would not sufficiently improve the noise reduction while a very wide kernel would slow down the convergence excessively especially for HYPR-AU-OSEM. Nevertheless, it can be observed from figure S4 that HYPR-AU-OSEM has the potential to increase CRC with a minimal increase in noise per iteration by applying a wider kernel in the HYPR operator. Therefore, 
improving the convergence without significantly increasing noise will be an important next step, and the optimal kernel size and number of iterations combination for HYPR-AU-OSEM will need to be determined.

When the HYPR operator was applied in the back-projection of HYPR-FB-OSEM, the filter kernel size was restricted to $3 \mathrm{~mm}$ for all cases in the simulations and $1.5 \mathrm{~mm}$ for the HRRT data to ensure accurate update factors can be obtained. When wider kernels were applied in the HYPR operator of the back-projection, higher noise increment per iteration, lower CRC's, and higher variation in resolution were observed (results not shown) likely due to that the update factor distribution across OSEM subsets is not as consistent as the activity distribution (i.e. the composite for the back-projection is likely to introduce bias in the update factors). As a result, an upper constraint in the kernel width needs to be applied in the HYPR operator of the back-projection for HYPR-FB-OSEM.

For the results presented in this work, the number of OSEM and HYPR-OSEM subsets was fixed at 16 throughout all evaluations. Although increasing the number of subsets can reduce the number of iterations to reach similar CRC's thus accelerating the reconstruction process, lower image quality might be obtained when there are insufficient counts within each subset. As expected, when using a higher number of subsets in HYPROSEM (results not shown), lower CRC's and higher noise were observed as a result of less accurate and less precise composite and target images due to insufficient counts within each subset, as compared to those obtained using the number of subsets presented in this work.

The HYPR operator can also be incorporated within algorithms that do not use subsets such as the MLEM. In a similar manner, a few MLEM updates are required to generate a composite with high resolution, but the number of the initial MLEM updates cannot be too high in order to avoid high frequency noise in the composite. The composite formation would 
be more flexible for MLEM than for OSEM as each MLEM update already utilizes all spatial or temporal data (for list-mode reconstruction). The way of summing the previous update images to form the composite as well as updating the composite would need to be optimized for MLEM in terms of noise reduction and efficient update of contrast. However, the drawback would be that the convergence of HYPR-MLEM methods is expected to be much slower than that of HYPR-OSEM methods.

The counting statistics chosen to perform the simulations in this study corresponded to a typical static imaging scan rather than a very low count case. Consequently, the number of counts within each OSEM subset was sufficient enough to reconstruct images without too much bias in contrast using the standard OSEM. Nevertheless, improvements in accuracy at the equivalent noise level and in precision were observed in general when comparing the proposed HYPR-OSEM to OSEM. For lower counting statistics cases such as reconstruction of a very short frame in dynamic studies, the proposed method showed greater improvements in accuracy and precision than those at high count level as demonstrated by the experimental phantom study. This is due to that OSEM reconstruction becomes less reproducible and more biased at low count level. The impact of HYPR-OSEM on dynamic frame-based reconstruction and the corresponding kinetic modeling parameters will need to be characterized with further investigations.

Incorporating the HYPR de-noising operator within the forward-projection and backprojection may seem similar to the image space resolution modeling approach (Reader et al. 2002, Rahmim et al. 2013). However, the two methods were designed for different purposes; i.e. the resolution modeling has been designed to improve accuracy, whereas the de-noising operator has been designed to improve the precision without altering the accuracy. Although it has been observed that the resolution modeling can reduce noise in voxel-level, it has been reported that the resolution modeling also worsens the variability in the ROI-level (Blinder $e t$ 
al. 2012, Rahmim et al. 2013). On the other hand, HYPR-OSEM methods have been demonstrated to improve the precision or reduce the variability in both voxel-level and ROIlevel CRCs. Furthermore, the de-noising operator does not introduce Gibb's artifacts as is the case for the resolution modeling (Blinder et al. 2012, Rahmim et al. 2013). Nevertheless, it would be beneficial to combine resolution modeling with HYPR-OSEM to improve both accuracy and precision, and additional explorations are required.

The current implementation of HYPR-OSEM can be considered as 'free' in that the composite is estimated only using the PET data without any assumptions about the tracer distribution and anatomical structures. It would be interesting to further investigate the possibility of incorporating, for example, an anatomical composite from MR or CT along with the functional composite from static and/or dynamic PET such that both anatomical structure boundaries and functional contrast can be preserved/enhanced with high SNR within the HYPR-OSEM frame work. On the other hand, one may consider this independent characteristic as an advantage since prior information can introduce bias in the reconstructed image (Wang and Qi 2009). In addition, the current implementation of HYPR-OSEM methods does not require high computational demands; i.e. the reconstruction time is about $10-20 \%$ longer than that of the original OSEM.

One limitation of HYPR post processing for dynamic imaging as reported previously (Cheng and Laforest 2012) is that HYPR cannot recover low activity regions filled with mostly zero value voxels due to low counting statistics in the short dynamic frame. By incorporating HYPR directly within the reconstruction of each dynamic frame, the number of zero value voxels within the object can be greatly reduced for all frames. Specifically, the voxel value after HYPR de-noising is zero only when the same voxel is zero in the composite or the filtered target image. As a result, HYPR-OSEM can further improve the accuracy of HYPR post processing for dynamic PET imaging especially for the quantitation in the 


\section{Acknowledgments} without sacrificing accuracy in terms of resolution and contrast recovery in frame-based PET reconstruction, thus showing great potential for applications which are sensitive to noise as well as applications which require low dose and/or scan time reduction. It has also been determined that HYPR-AU-OSEM is the more effective form than HYPR-F(B)-OSEM in terms of accuracy and precision. In addition, the proposed method can be applied to improve the image quality of any frame-based iterative reconstruction for both SPECT and PET without any prior information about the imaging task. Future works include improving the convergence and determining optimal reconstruction parameters for HYPR-AU-OSEM, combining HYPR-AU-OSEM with other system models such as resolution modeling, and investigating the impact of HYPR-AU-OSEM on clinical applications.

This work was supported by the Netherlands Organization for Health Research and Development IMDI grant 104002006, CRUK \& EPSRC Cancer Imaging Centre in Cambridge and Manchester (C8742/A18097), and Brain Canada PT 64457. It was presented in part at the annual Society of Nuclear Medicine and Molecular Imaging Meeting, San Diego, CA, June 2016 and at the IEEE Medical Imaging Conference, Strasbourg, France, November 2016.

\section{References}


Bai, B., Q. Li, and R. M. Leahy (2013). "Magnetic resonance-guided positron emission tomography image reconstruction." Seminars in Nuclear Medicine 43(1): 30-44.

Blinder, S. A. L., K. Dinelle and V. Sossi (2012). "Scanning rats on the high resolution research tomograph (HRRT): A comparison study with a dedicated micro-PET." Medical Physics 39: 5073-5083.

Boellaard, R., et al. (2010). "FDG PET and PET/CT: EANM procedure guidelines for tumour PET imaging: version 1.0." Eur J Nucl Med Mol Imaging 37(1): 181-200.

Cheng, J. C. and R. Laforest (2012). Evaluation of HYPR de-noising with MAP reconstruction in small animal PET imaging. Nuclear Science Symposium and Medical Imaging Conference (NSS/MIC), 2012 IEEE.

Christian, B. T., N. T. Vandehey, J. M. Floberg and C. A. Mistretta (2010). "Dynamic PET denoising with HYPR processing." J Nucl Med 51(7): 1147-1154.

Floberg, J. M., C. A. Mistretta, J. P. Weichert, L. T. Hall, J. E. Holden and B. T. Christian (2012). "Improved kinetic analysis of dynamic PET data with optimized HYPR-LR." Med Phys 39(6): 3319-3331.

Fowler, K. J., N. M. Maughan, R. Laforest, N. E. Saad, A. Sharma, J. Olsen, ... P. J. Parikh (2016). "PET/MRI of Hepatic 90Y Microsphere Deposition Determines Individual Tumor Response." Cardiovascular and Interventional Radiology 39: 855-864.

Hudson, H. M. and R. S. Larkin (1994). "Accelerated image reconstruction using ordered subsets of projection data." IEEE Transactions on Medical Imaging 13(4): 601-609.

de Jong, H. W., F. H. van Velden, R. W. Kloet, F. L. Buijs, R. Boellaard and A. A. Lammertsma (2007). "Performance evaluation of the ECAT HRRT: an LSO-LYSO double layer high resolution, high sensitivity scanner." Physics in Medicine and Biology 52(5): $1505-1526$.

Johnson, K. M., J. Velikina, Y. Wu, S. Kecskemeti, O. Wieben and C. A. Mistretta (2008). "Improved Waveform Fidelity Using Local HYPR Reconstruction (HYPR LR)." Magnetic resonance in medicine : official journal of the Society of Magnetic Resonance in Medicine / Society of Magnetic Resonance in Medicine 59(3): 456-462.

Lartizien, C., J. B. Aubin and I. Buvat (2010). "Comparison of Bootstrap Resampling Methods for 3-D PET Imaging." IEEE Transactions on Medical Imaging 29: 1442-54.

Markiewicz, P. J., A. J. Reader and J. C. Matthews (2015). "Assessment of bootstrap resampling performance for PET data." Physics in Medicine and Biology 60: 279-99.

NEMA (2007). Performance measurements of positron emission tomographs. Rosslyn, VA., NEMA Standards Publication NU 2-2007: 26-33. 
Nyflot, M. J., F. Yang, D. Byrd, S. R. Bowen, G. A. Sandison and P. E. Kinahan (2015). "Quantitative radiomics: impact of stochastic effects on textural feature analysis implies the need for standards." Journal of Medical Imaging 2(4): 041002-041002.

Rahmim, A., J. Qi, V. Sossi (2013). "Resolution modeling in PET imaging: Theory, practice, benefits, and pitfalls." Medical Physics 40(6): 064301.

Rahmim, A., J. Tang and H. Zaidi (2009). "Four-dimensional (4D) image reconstruction strategies in dynamic PET: Beyond conventional independent frame reconstruction." Medical Physics 36(8): 3654-3670.

Reader, A. J., P. J. Julyan, H. Williams, D. L. Hastings and J. Zweit (2002). "EM álgorithm resolution modeling by image-space convolution for PET reconstruction." IEEE Nuclear Science Symposium Conference Record 2: 1221-1225.

Reader, A. J. and J. Verhaeghe (2014). "4D image reconstruction for emission tomography." Physics in Medicine and Biology 59(22): R371.

Vaissier, P. E. B., M. C. Goorden, A. B. Taylor and F. J. Beekman (2013) "Fast CountRegulated OSEM Reconstruction With Adaptive Resolution Recovery." IEEE Transactions on Medical Imaging 32(12): 2250-2261.

Vriens, D., J. A. Disselhorst, W. J. G. Oyen, L.-F. de Geus-Oei and E. P. Visser (2012). "Quantitative Assessment of Heterogeneity in Tumor Metabolism Using FDG-PET." International Journal of Radiation Oncology*Biology*Physics 82(5): e725-e731.

Wang, G. and J. Qi (2009). "Analysis of Penalized Likelihood Image Reconstruction for Dynamic PET Quantification." IEEE Trans Med Imaging 28(4): 608-620. 